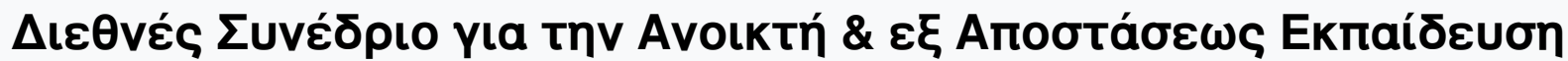

Tóp. 6, Ap. 2B (2011)

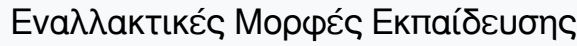

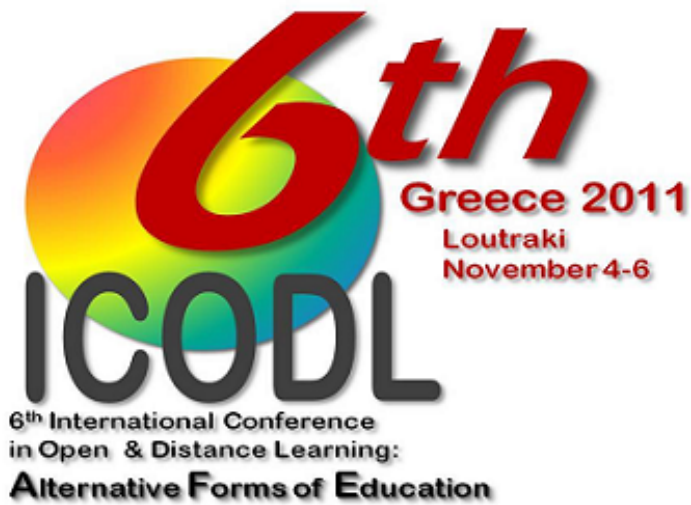

TOMOE B

PART / MEPOE B

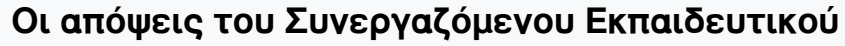

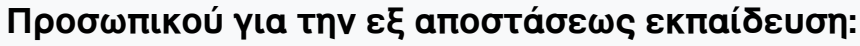

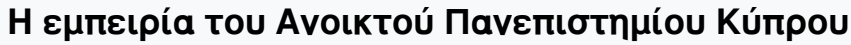

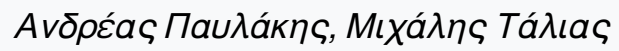

doi: $10.12681 /$ icodl. 668 


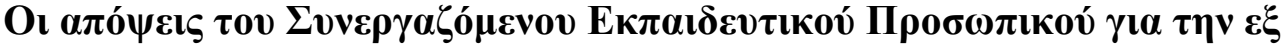

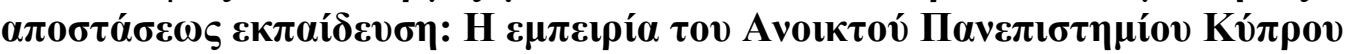

\section{The adjunct professors' opinions for the distance education: the case of Open University of Cyprus}

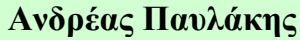

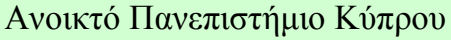 \\ $\Lambda \varepsilon ́ \kappa \tau о \rho \alpha \varsigma$ \\ a.pavlakis@ouc.ac.cy
}

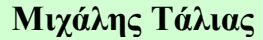

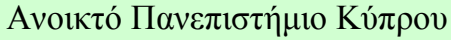

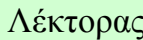 \\ michael.talias@ouc.ac.cy
}

\begin{abstract}
This paper focuses on the views and the role of adjunct professors of the Open University of Cyprus with regard to teaching methodology of the Open and distance learning pragmatism and the use of technology and administrative support.

Specifically, our findings suggest that the adjunct professors perceive as more important and substantial their role of the open and distance learning than the conventional procedure. They also trust and use new technologies in carrying out their duties.

Additionally, it is shown that they were satisfied by the administrative support and their educational environment at the Open University to a higher degree than the conventional university environment.

Furthermore, several of their positions on various aspects of the methodology differ substantially between professors of different programs.
\end{abstract}

\section{Пєрі́ $\eta \psi \eta$}

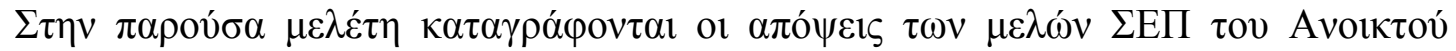

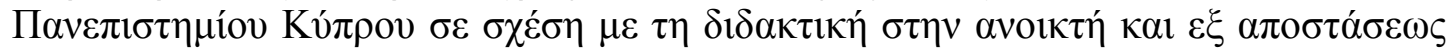

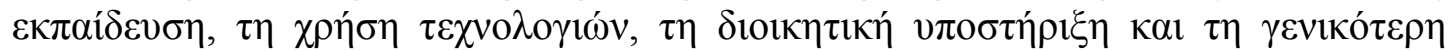

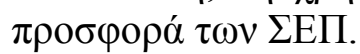

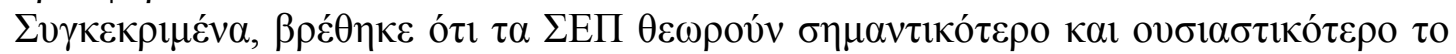

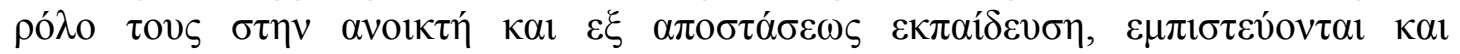

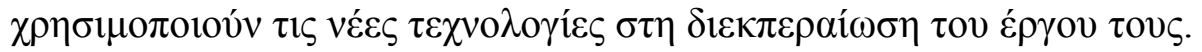

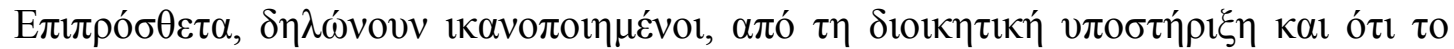

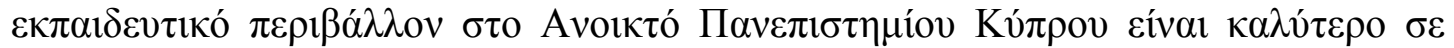
$\sigma \chi \varepsilon \dot{\varepsilon} \eta \mu \varepsilon \tau \eta \sigma u \mu \beta \alpha \tau \imath \kappa \eta ́ ~ \varepsilon \kappa \pi \alpha i ́ \delta \varepsilon v \sigma \eta$.

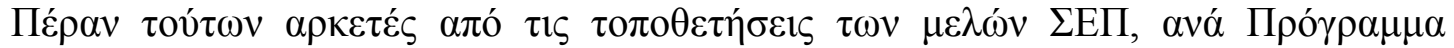

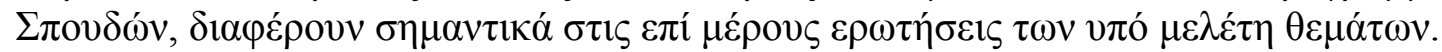

Key-words: Open University of Cyprus, use of technology, distance learning, adjunct professors opinions

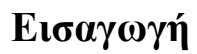

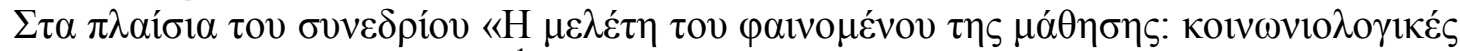

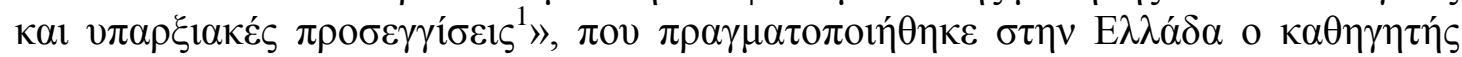

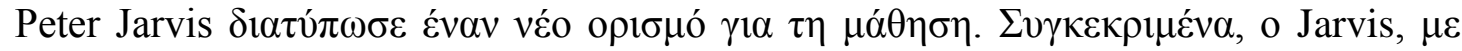

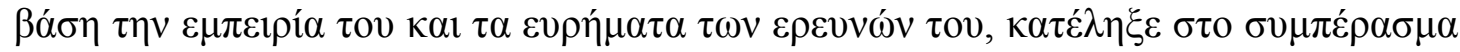


ó $\tau$ "Learning is the process whereby living organisms consciously experience and

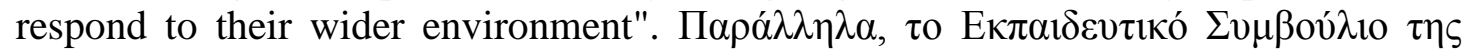

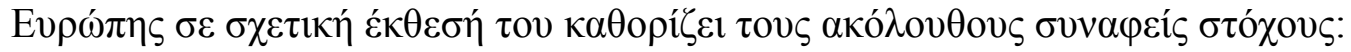

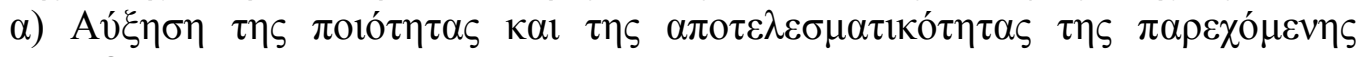

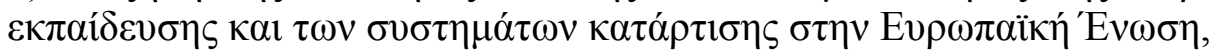

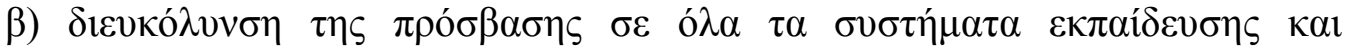

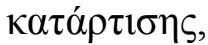

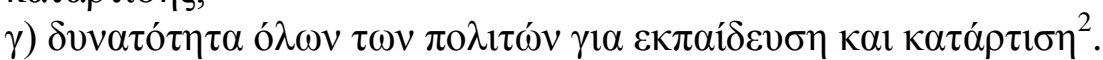

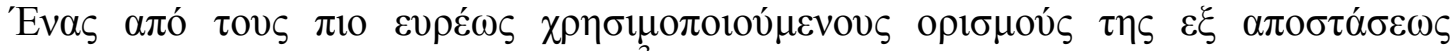

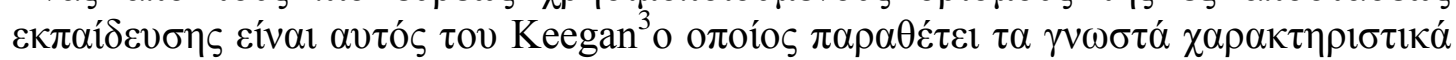

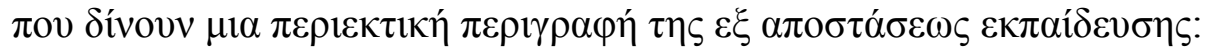

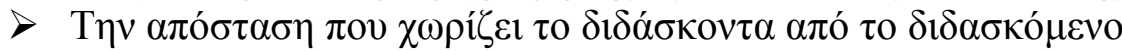

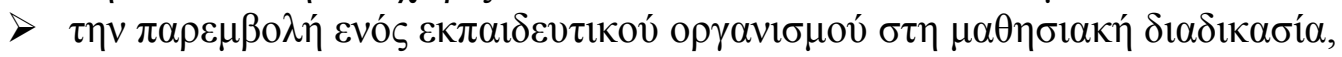

$>\tau \eta \chi \rho \eta \dot{\sigma \eta} \tau \varepsilon \chi v i \kappa \omega ́ v ~ \mu \varepsilon ́ \sigma \omega v$,

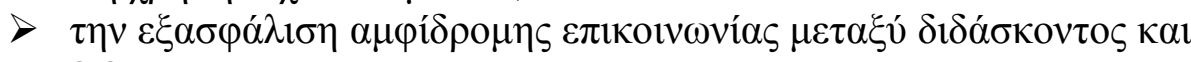
$\delta 1 \delta \alpha \sigma \kappa о \mu \varepsilon ́ v o v$,

$>\tau \eta \delta v v \alpha \tau o ́ \tau \eta \tau \alpha \sigma v v \alpha v \tau \eta ́ \sigma \varepsilon \omega v \delta 1 \delta \alpha \sigma \kappa o ́ v \tau \omega v \kappa \alpha l \delta 1 \delta \alpha \sigma \kappa o \mu \varepsilon ́ v \omega v$.

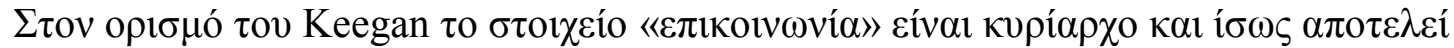

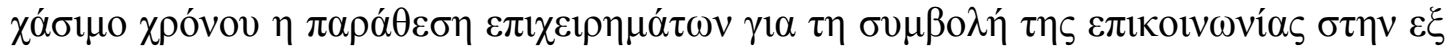

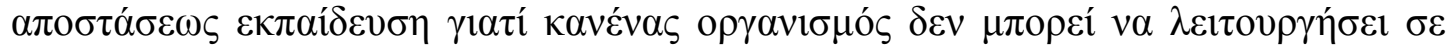

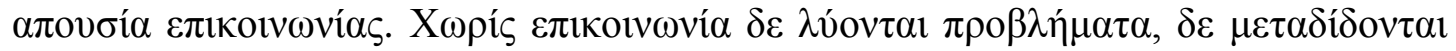

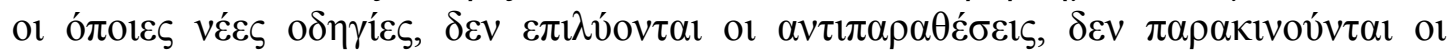

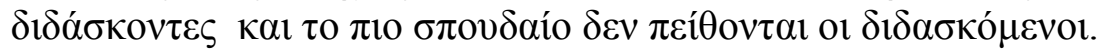

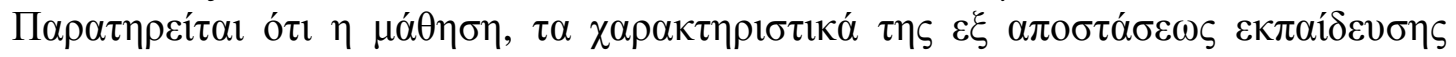

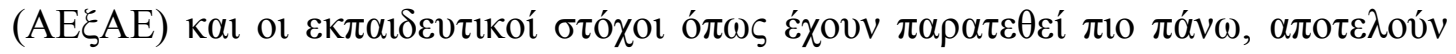

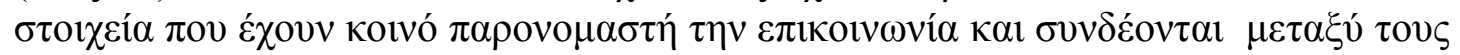

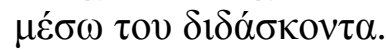

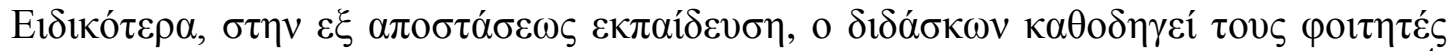

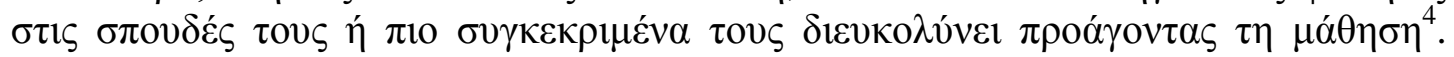

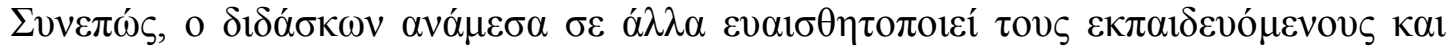

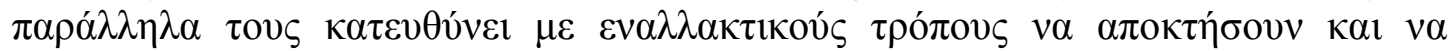
$\alpha \varphi$ o

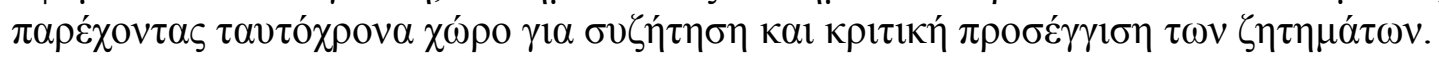

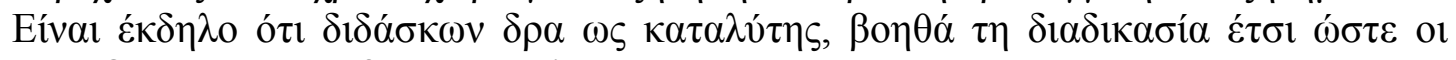

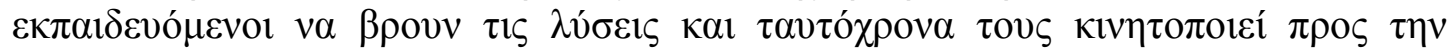

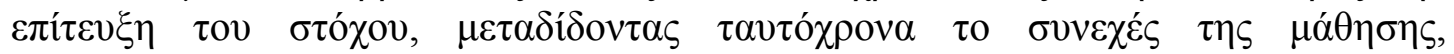

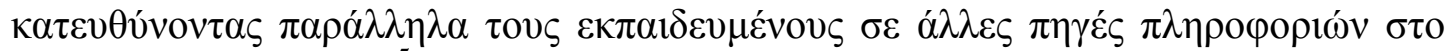

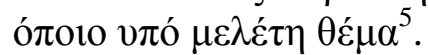

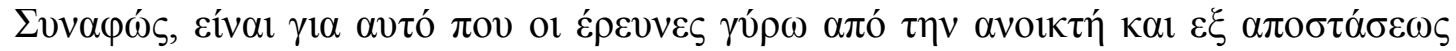

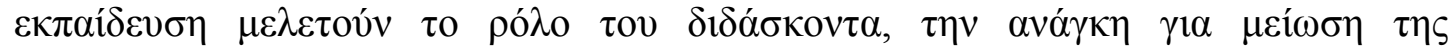

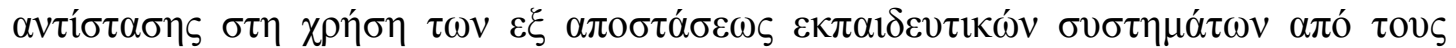

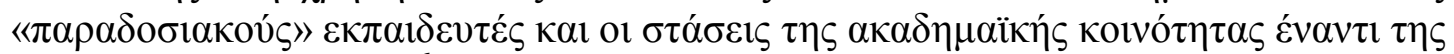

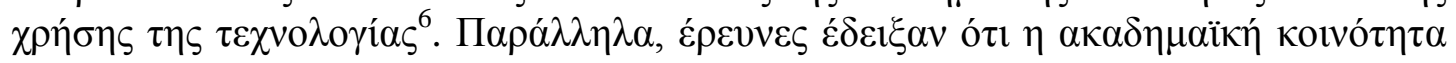

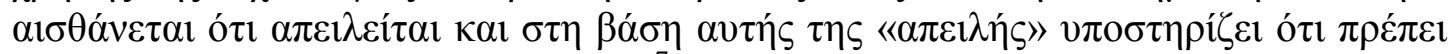

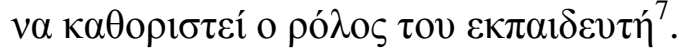

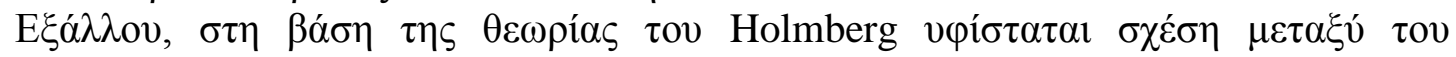

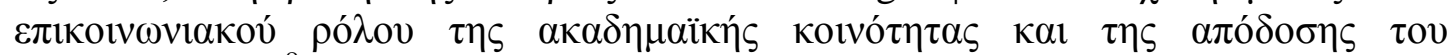

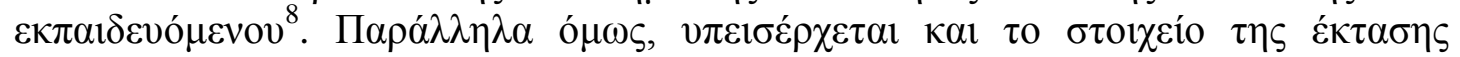

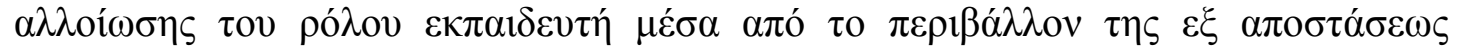

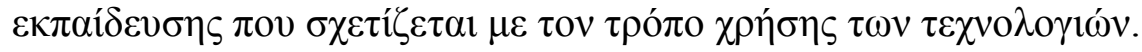




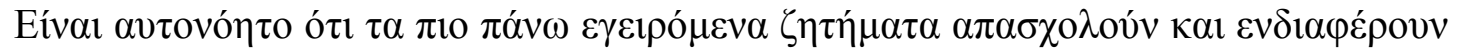

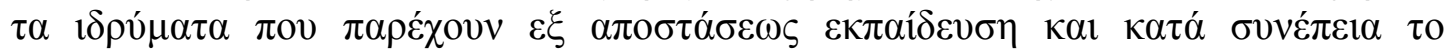

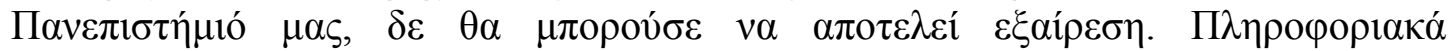

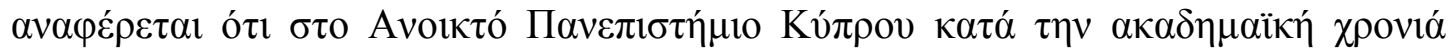
$2010-11, \delta \eta \lambda \alpha \delta \eta \dot{~ \tau o v ~ \pi \varepsilon ́ \mu \pi \tau o ~ \chi \rho o ́ v o ~ \lambda \varepsilon ı \tau o v \rho \gamma i ́ a s ~ \tau o v, ~ v \pi \eta \rho \varepsilon \tau o v ́ \sigma \alpha v ~} 115 \mu \varepsilon \dot{\lambda} \eta$

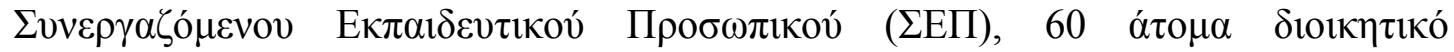

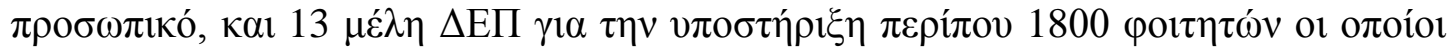

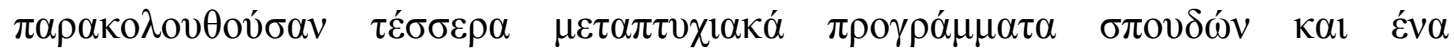

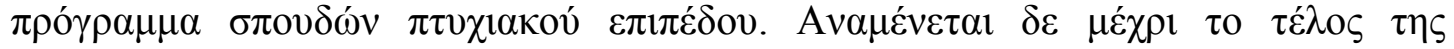

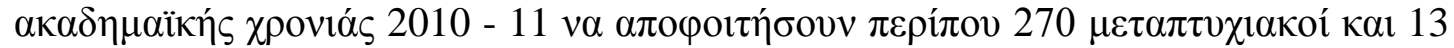

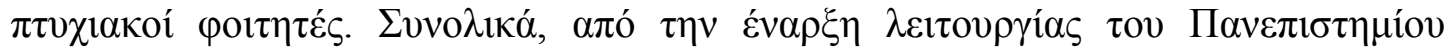

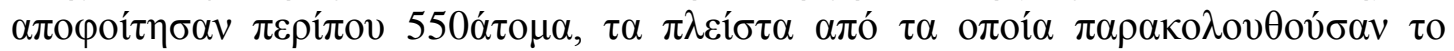

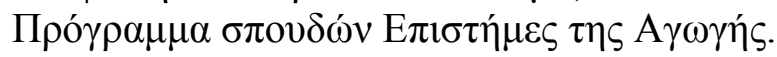

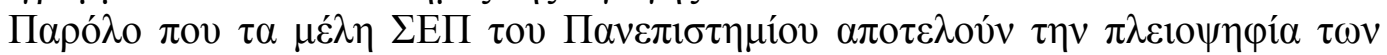

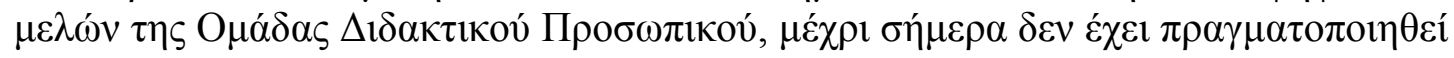

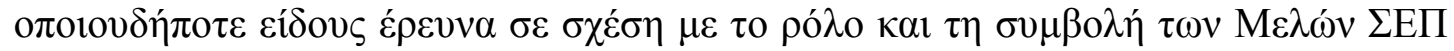

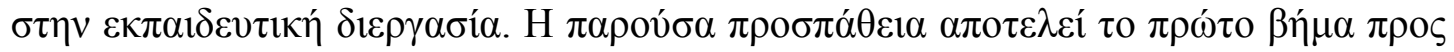

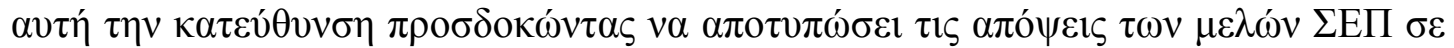

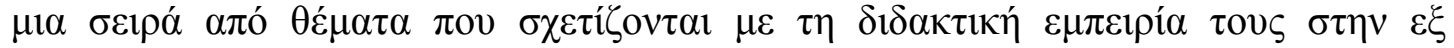

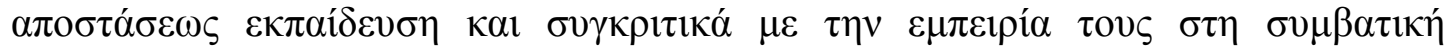
$\varepsilon \kappa \pi \alpha i ́ \delta \varepsilon v \sigma \eta$.

\section{$\mathrm{ME \Theta O} \triangle \mathrm{O} \Lambda \mathrm{O} \Gamma \mathrm{IA} T \mathrm{TH} \Sigma$ EPEYNA $\Sigma$}

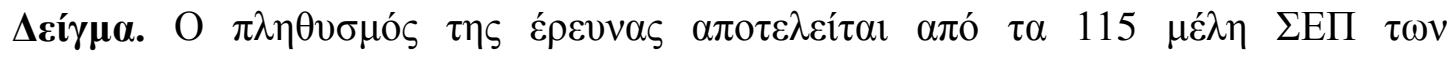

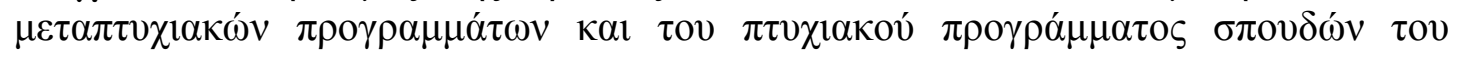

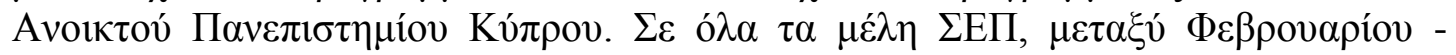

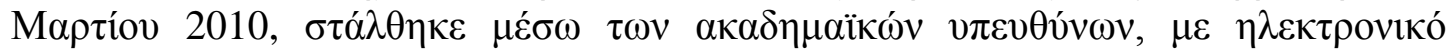

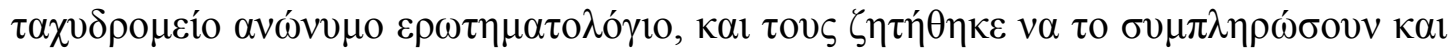

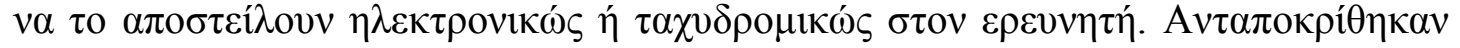

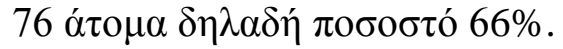

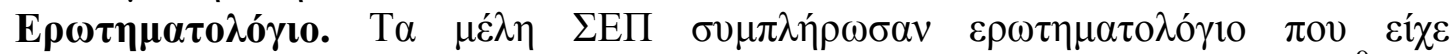

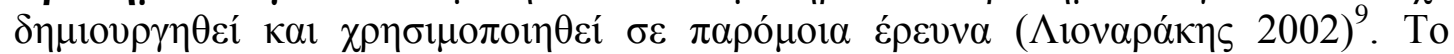

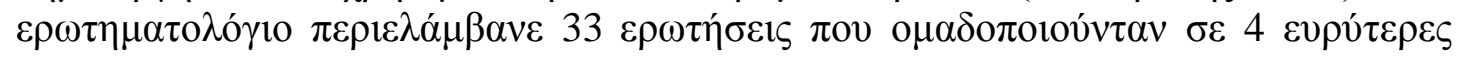
$\mu \varepsilon \tau \alpha \beta \lambda \eta \tau \dot{\varepsilon} \varsigma$ :

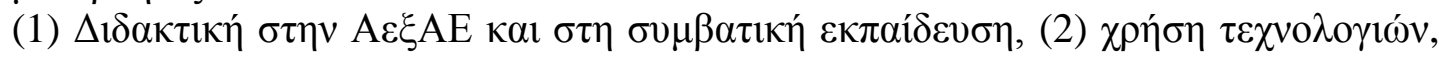

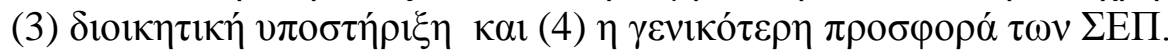

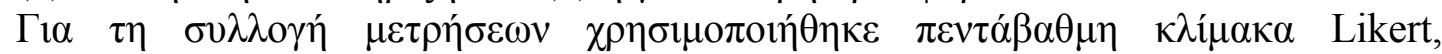

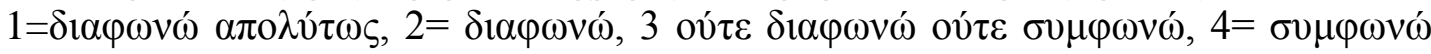

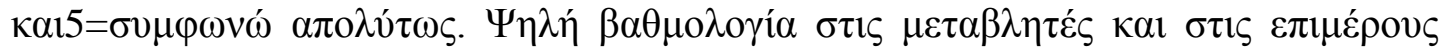

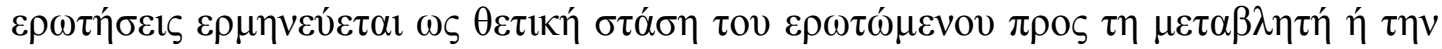

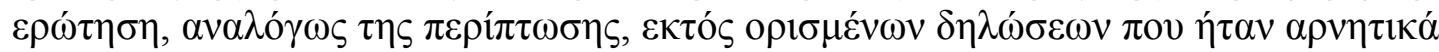
$\delta 1 \alpha \tau v \pi \omega \mu \varepsilon ́ v \varepsilon \varsigma$.

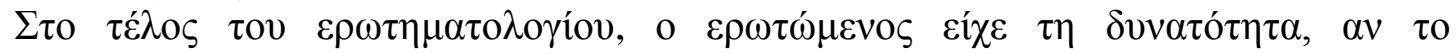

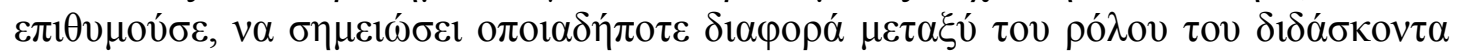

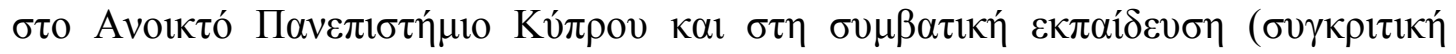

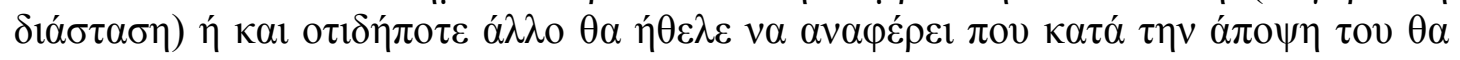

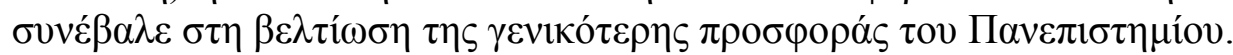

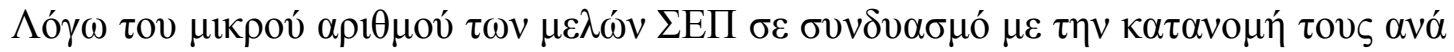

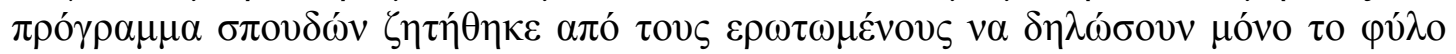




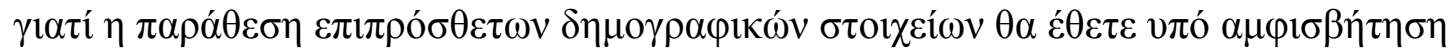

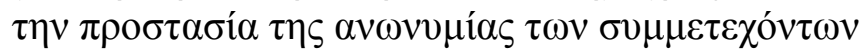

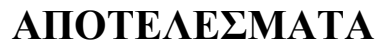

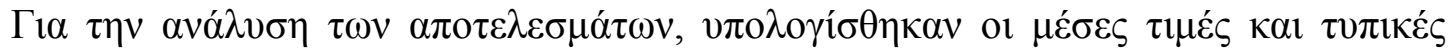

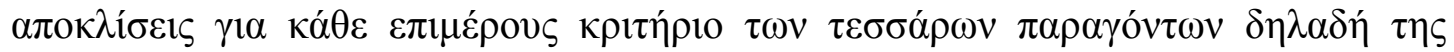

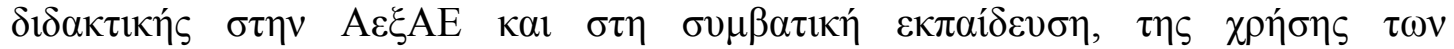

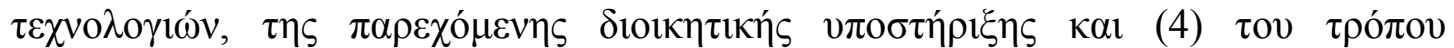

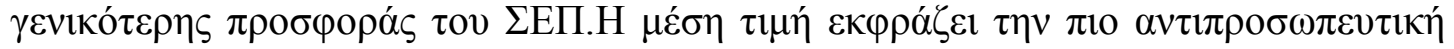

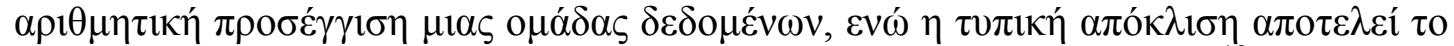

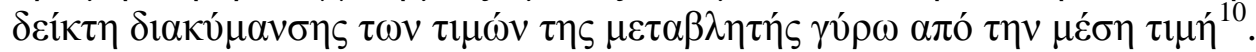

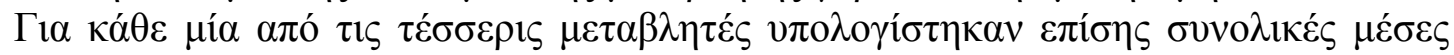

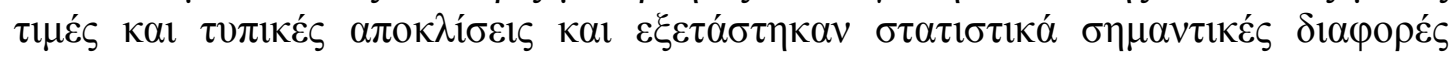

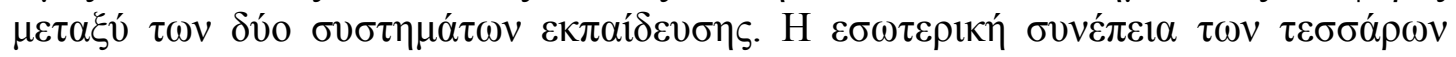

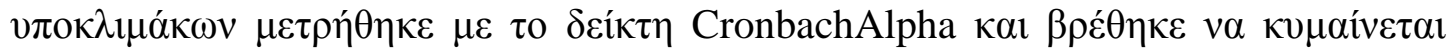

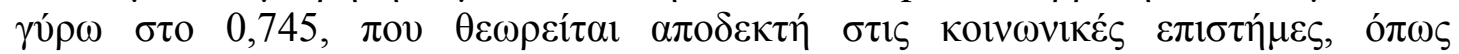

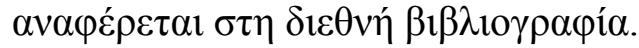

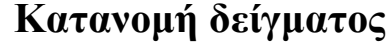

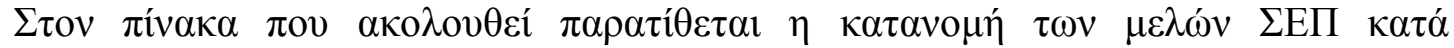

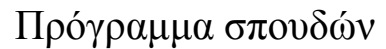

Пívakas 1

A

\begin{tabular}{|c|c|c|c|c|c|}
\hline & \multicolumn{5}{|c|}{ 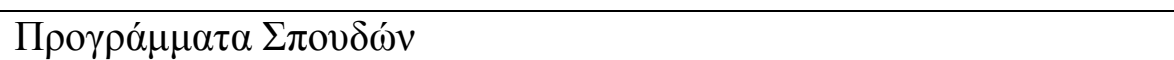 } \\
\hline & 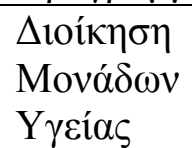 & 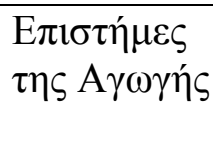 & 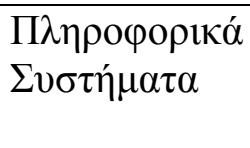 & $\mathrm{M} \Delta \mathrm{E}$ & 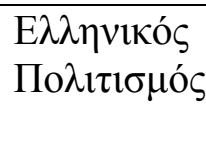 \\
\hline $\begin{array}{l}\text { Apır } \theta \text { ós } \\
\Sigma \text { ЕП }\end{array}$ & $\begin{array}{l}15 \\
(17)^{*}\end{array}$ & $\begin{array}{l}13 \\
(21)\end{array}$ & $\begin{array}{l}11 \\
(13)\end{array}$ & $\begin{array}{l}18 \\
(31)\end{array}$ & $\begin{array}{l}19 \\
(33)\end{array}$ \\
\hline
\end{tabular}

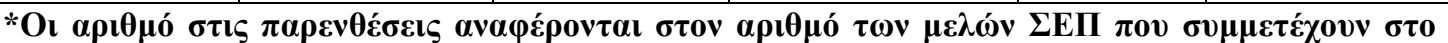

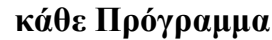

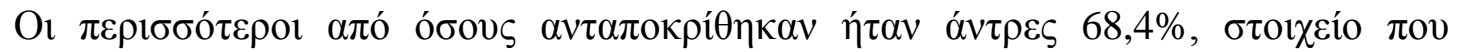

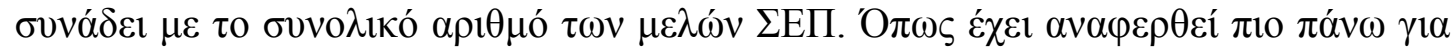

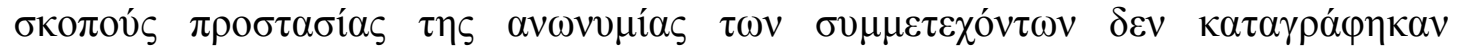

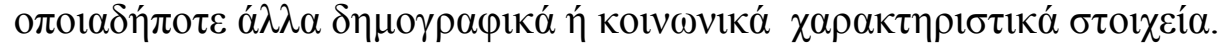

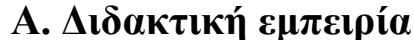

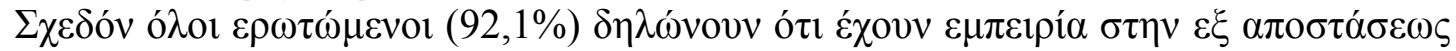

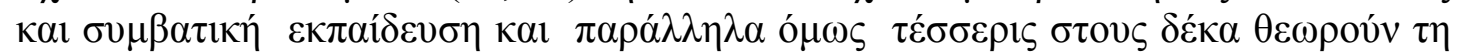

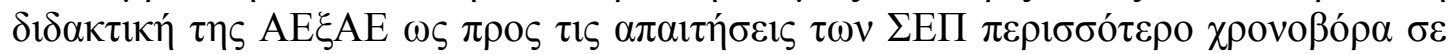

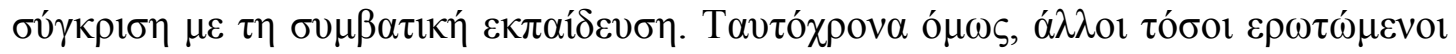

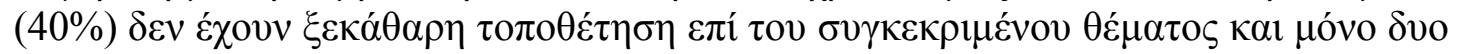

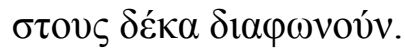

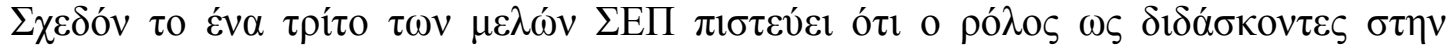

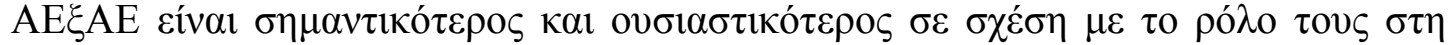

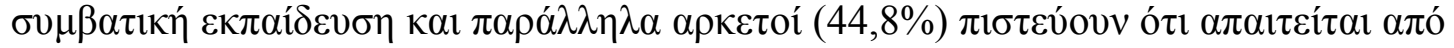

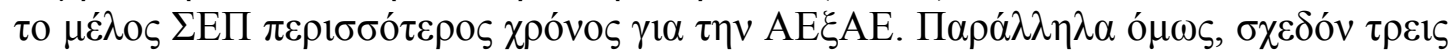

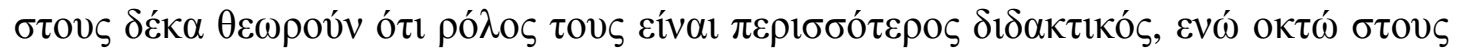




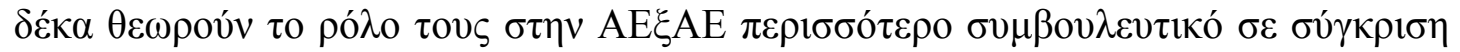
$\mu \varepsilon \tau \eta \sigma v \mu \beta \alpha \tau 1 \kappa \eta ́$.

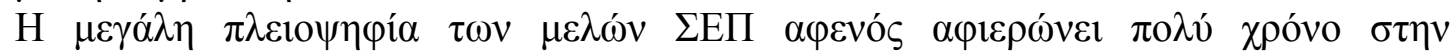

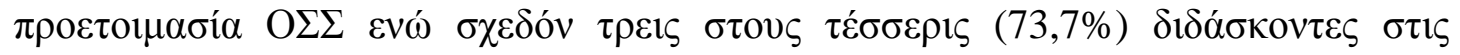

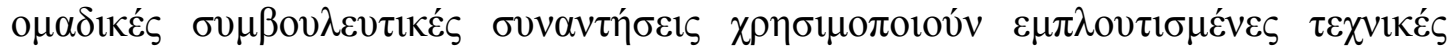

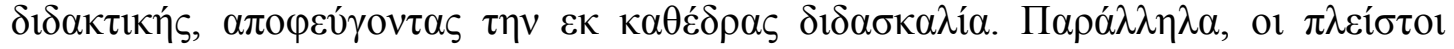

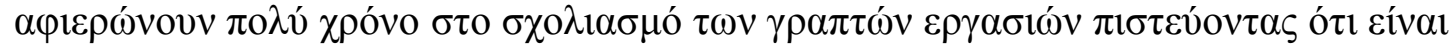

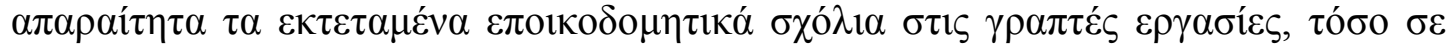

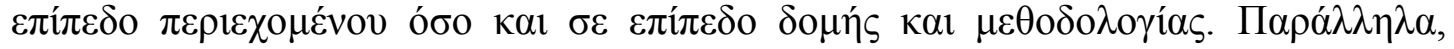

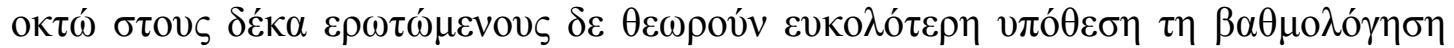

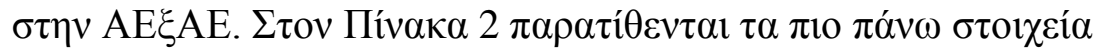

Пívakas 2

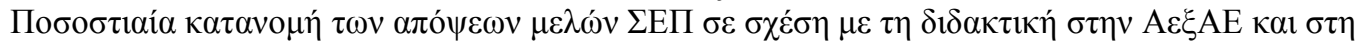
$\sigma 0 \mu \beta \alpha \tau \iota \kappa \eta ́ ~ \varepsilon \kappa \pi \alpha i ́ \delta \varepsilon v \sigma \eta$

\begin{tabular}{|c|c|c|}
\hline$\Delta \eta \lambda \omega ́ \sigma \varepsilon 1 \varsigma$ & $\Delta \iota \alpha \varphi \omega v \omega ́$ & $\Sigma v \mu \varphi \omega v \omega ́$ \\
\hline 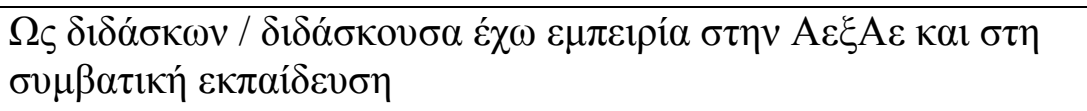 & 2,6 & 92,1 \\
\hline 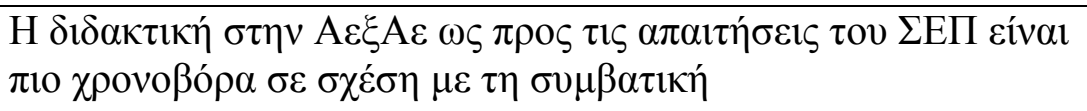 & 18,6 & 41,3 \\
\hline 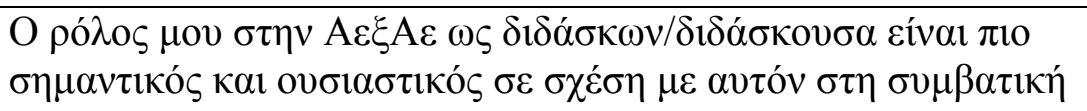 & 23,7 & 32,9 \\
\hline 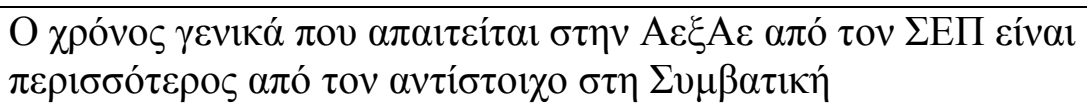 & 25,0 & 44,8 \\
\hline 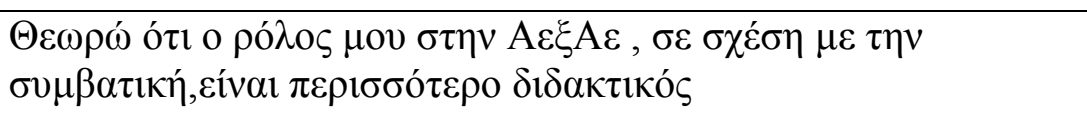 & 44,6 & 28,4 \\
\hline 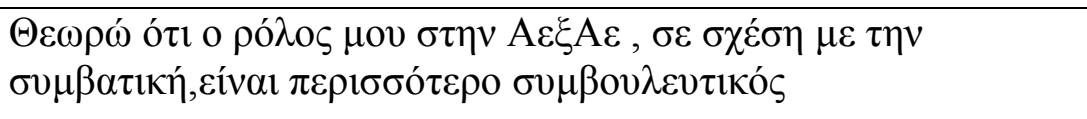 & 8,0 & 82,7 \\
\hline 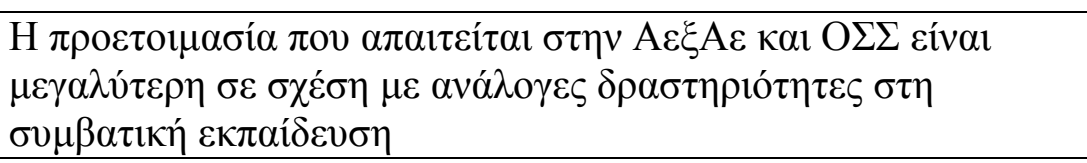 & 9,5 & 63,5 \\
\hline 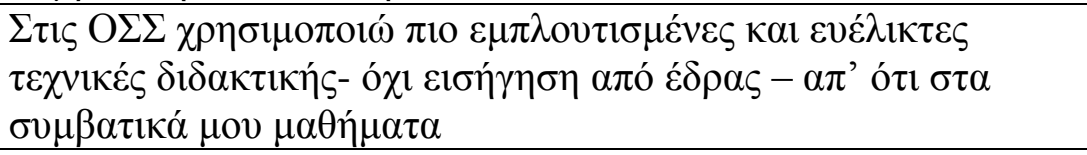 & 10,5 & 73,7 \\
\hline 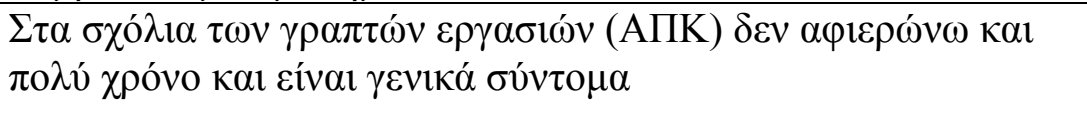 & 89,5 & 5,2 \\
\hline 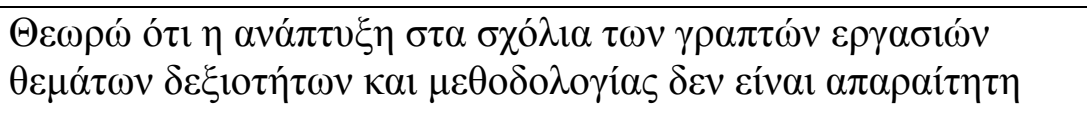 & 88,10 & 3,9 \\
\hline 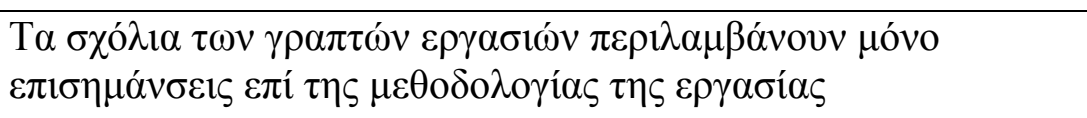 & 90,8 & 1,3 \\
\hline 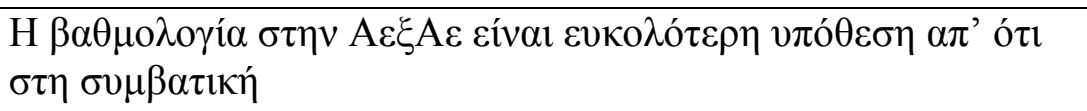 & 76,4 & 2,6 \\
\hline
\end{tabular}

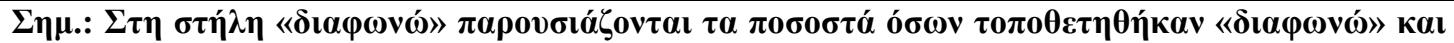

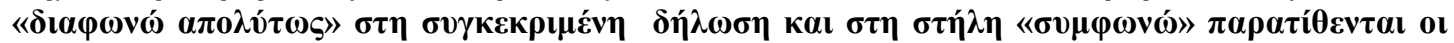

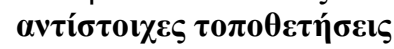




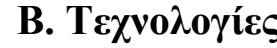

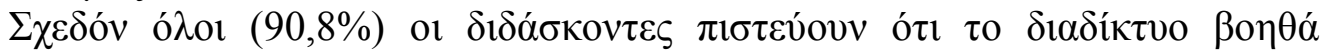

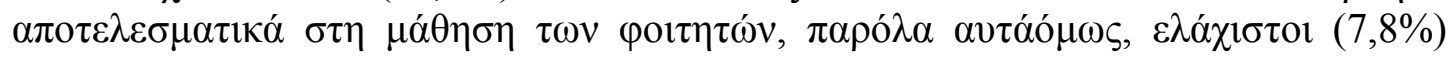

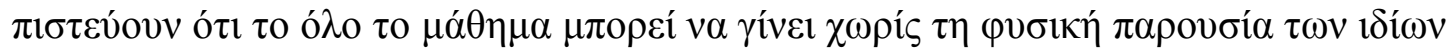

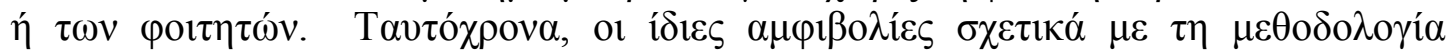

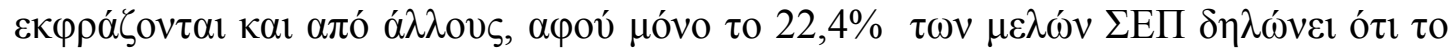

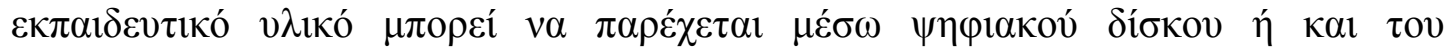

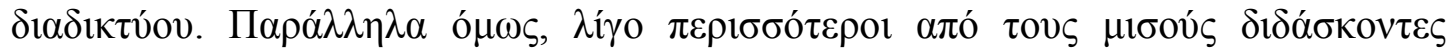

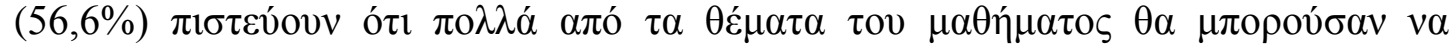

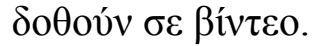

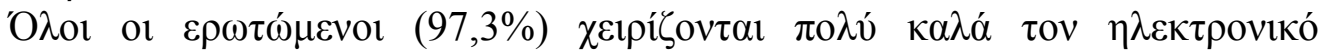

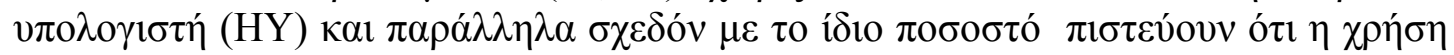

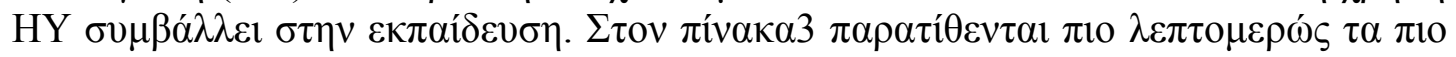

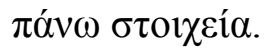

Пívakaৎ 3

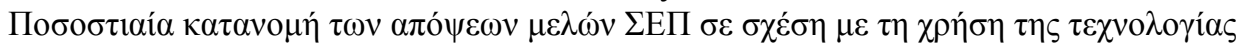

\begin{tabular}{|c|c|c|}
\hline$\Delta \eta \lambda \omega ́ \sigma \varepsilon 1 \varsigma$ & $\Delta 1 \alpha \varphi \omega v \omega ́$ & $\Sigma v \mu \varphi \omega v \omega ́$ \\
\hline 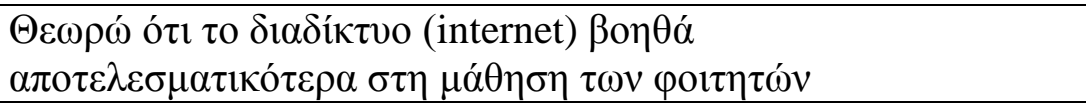 & 2,6 & 90,8 \\
\hline 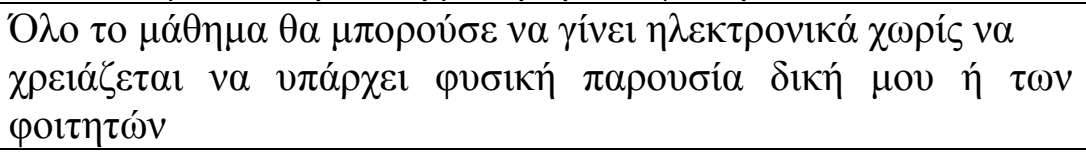 & 75,0 & 7,6 \\
\hline 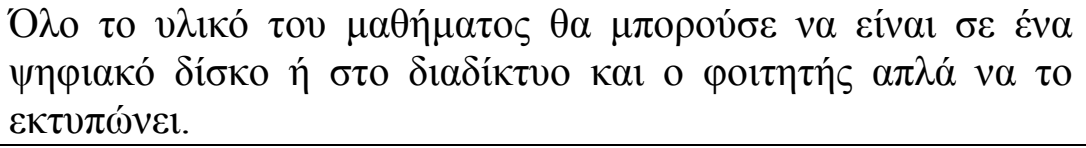 & 57,90 & 22,4 \\
\hline 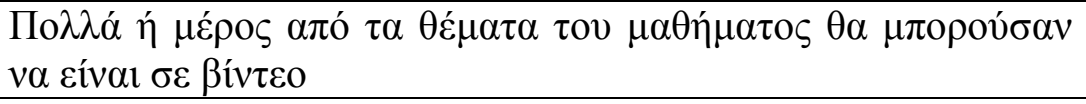 & 18,40 & 56,6 \\
\hline 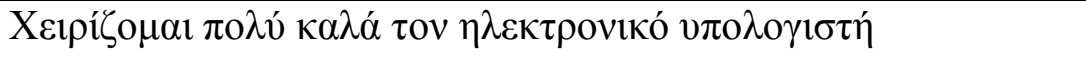 & - & 97,3 \\
\hline 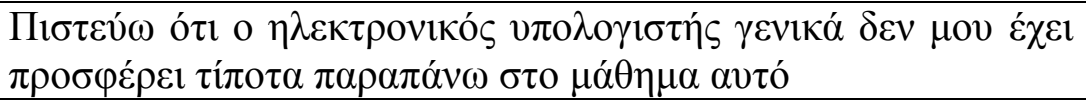 & 96,0 & 1,3 \\
\hline
\end{tabular}

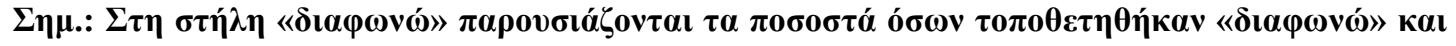

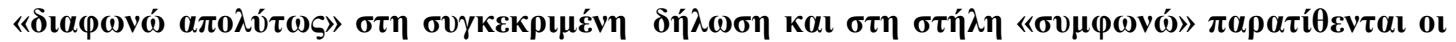

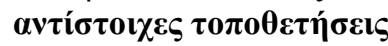

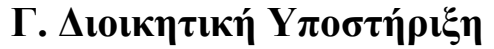

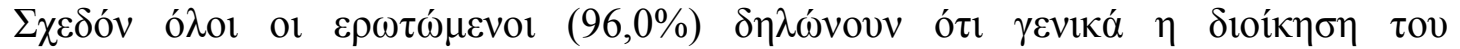

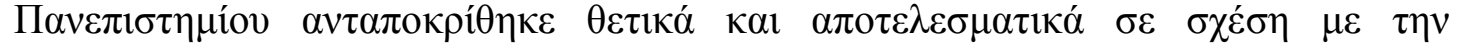

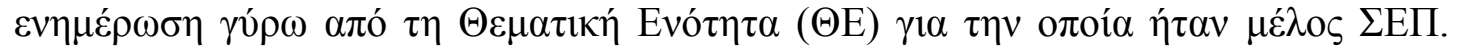

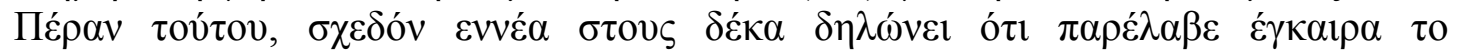
$\varepsilon \kappa \pi \alpha \imath \delta \varepsilon v \tau \imath$ เ

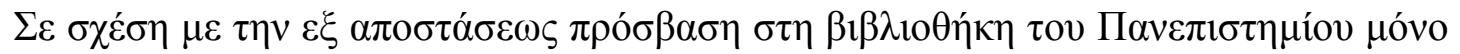

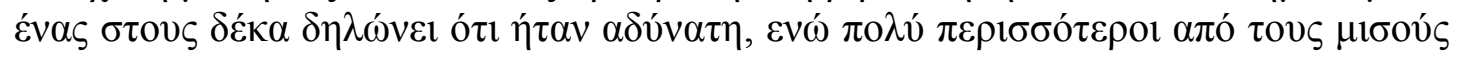

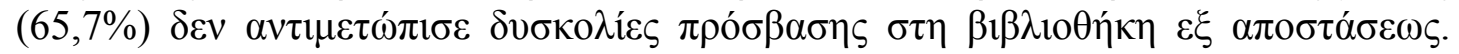

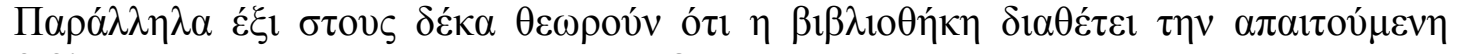

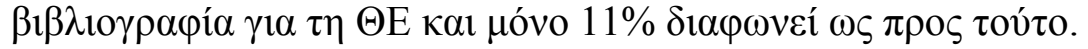

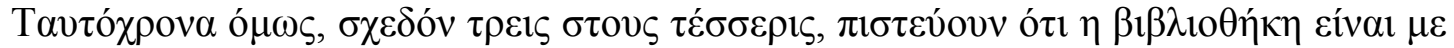

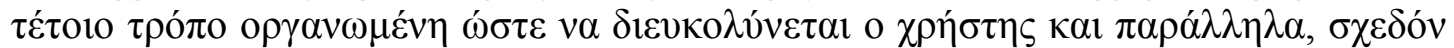

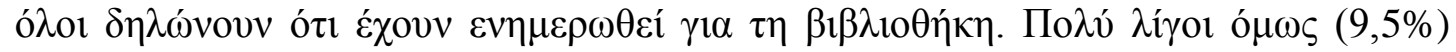




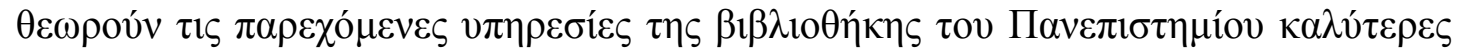

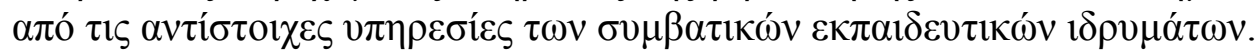

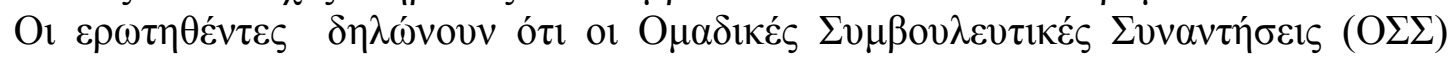

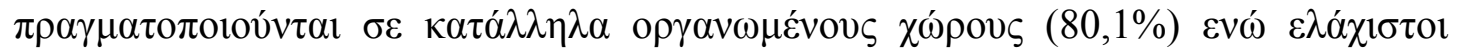

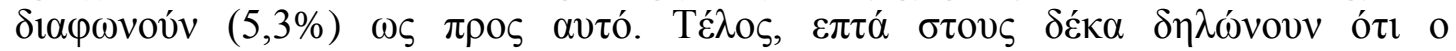

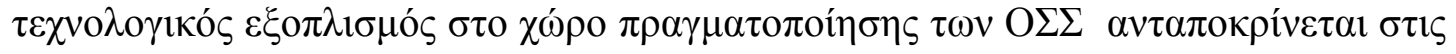

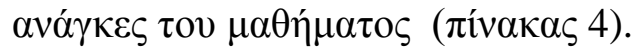

Пívakas 4

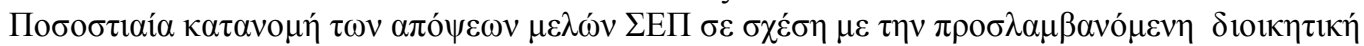

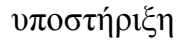

\begin{tabular}{|c|c|c|}
\hline$\Delta \eta \lambda \omega ́ \sigma \varepsilon 1 \varsigma$ & $\Delta 1 \alpha \varphi \omega v \omega ́$ & $\Sigma v \mu \varphi \omega v \omega ́$ \\
\hline 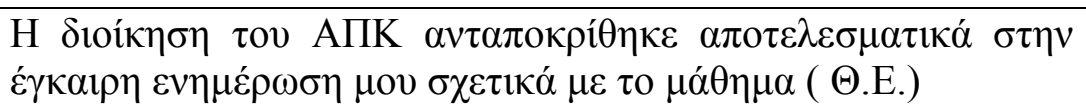 & - & 96,0 \\
\hline 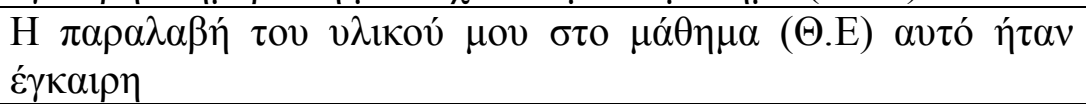 & 4,10 & 87,8 \\
\hline 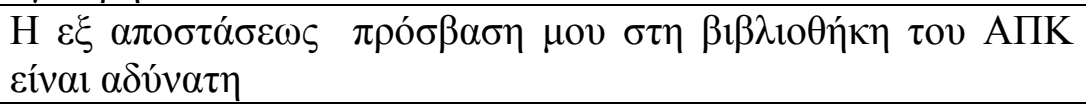 & 65,70 & 11,0 \\
\hline 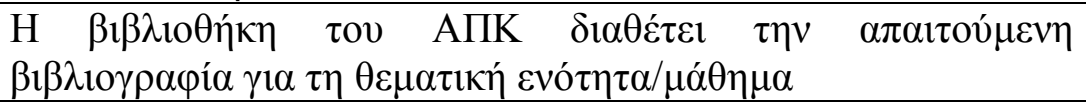 & 11,0 & 60,3 \\
\hline 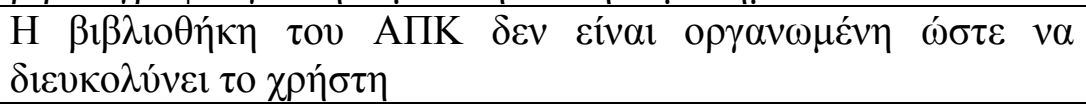 & 73,90 & 7,2 \\
\hline 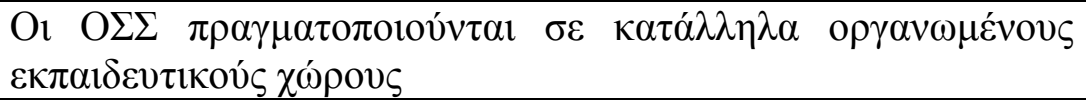 & 5,3 & 80,0 \\
\hline 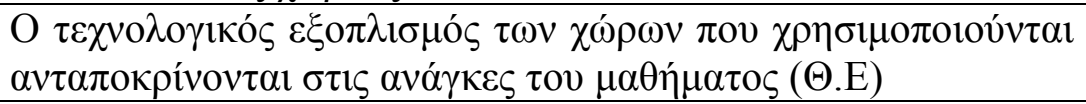 & 12,00 & 73,3 \\
\hline 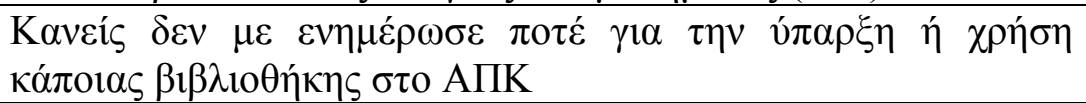 & 94,60 & 2,8 \\
\hline 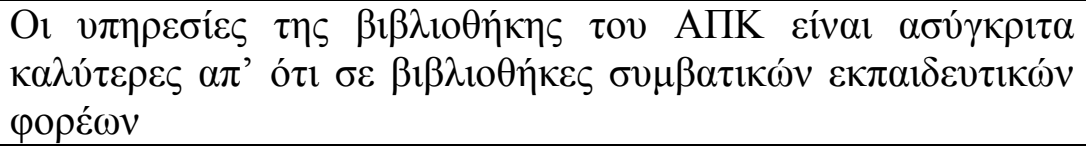 & 28,70 & 9,5 \\
\hline
\end{tabular}

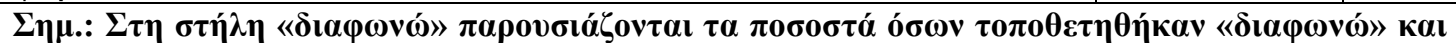

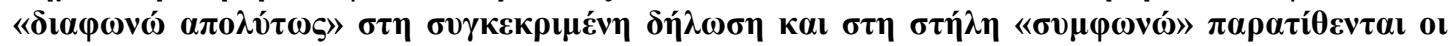

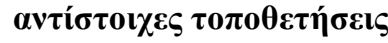

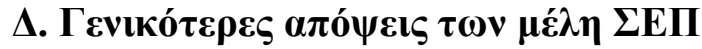

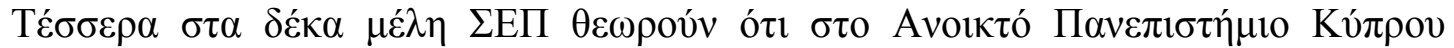

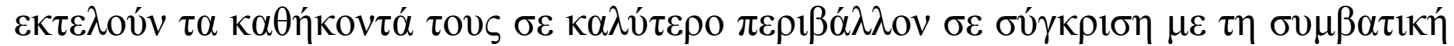

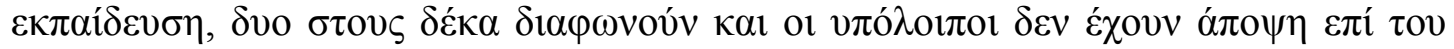

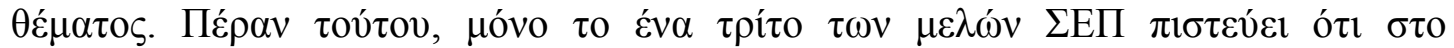

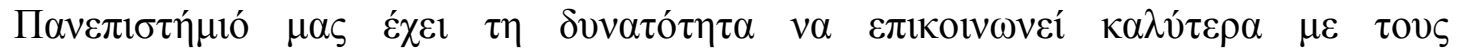

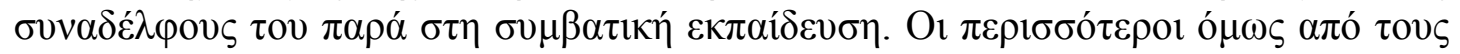
$\varepsilon \rho \omega \tau \omega ́ \mu \varepsilon v o v \varsigma ~(54,8 \%) \pi \imath \tau \varepsilon v ́ o v v$ ó

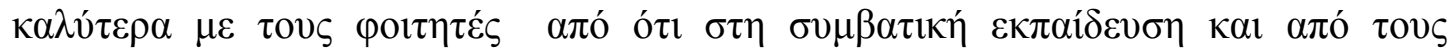

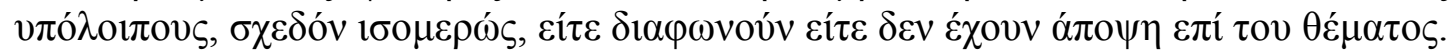

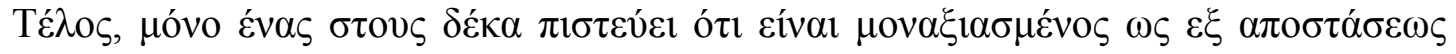

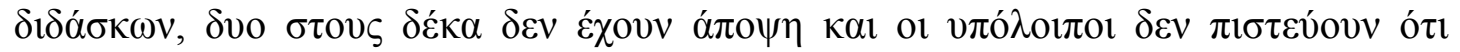

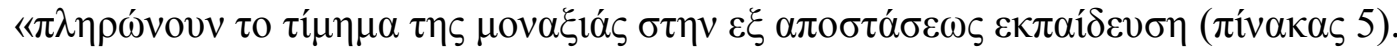




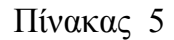

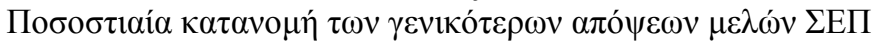

\begin{tabular}{|c|c|c|}
\hline$\Delta \eta \lambda \omega ́ \sigma \varepsilon 1 \varsigma$ & $\Delta ı \alpha \varphi \omega v \omega ́$ & $\Sigma v \mu \varphi \omega v \omega ́$ \\
\hline 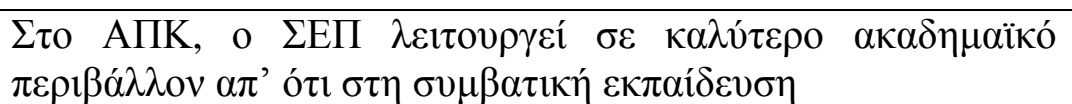 & 20,0 & 40,0 \\
\hline 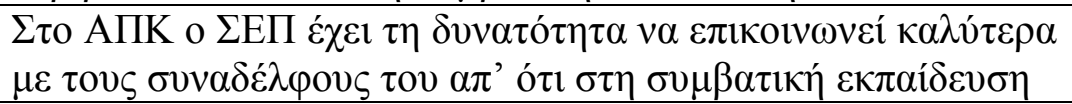 & 25,4 & 33,3 \\
\hline 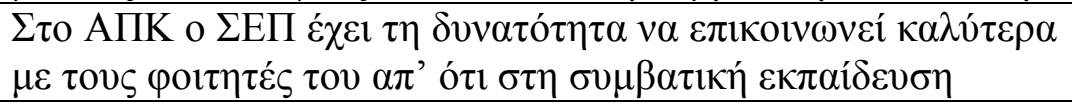 & 23,3 & 54,8 \\
\hline 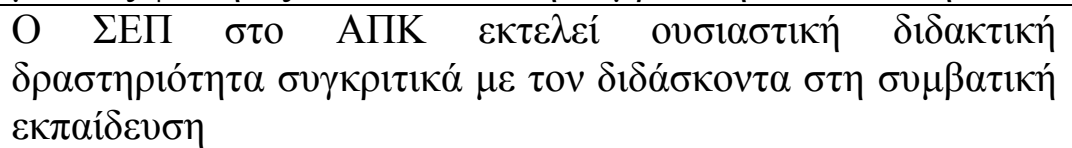 & 25,4 & 28,0 \\
\hline 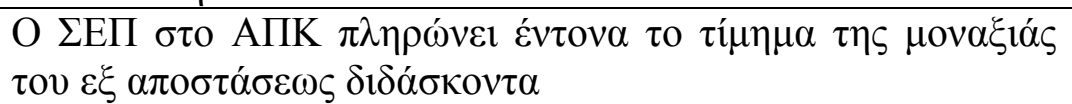 & 66,7 & 10,7 \\
\hline
\end{tabular}

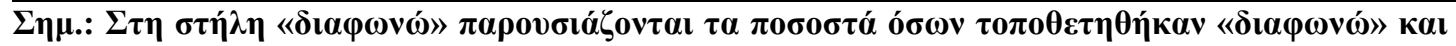

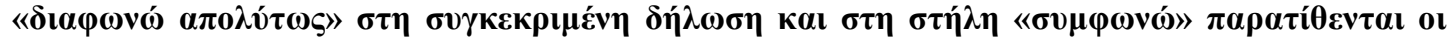

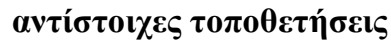

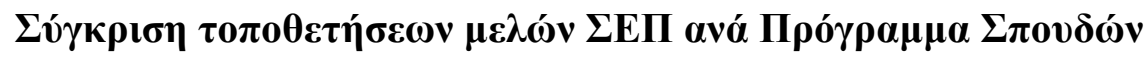

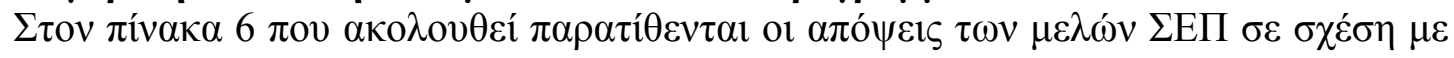

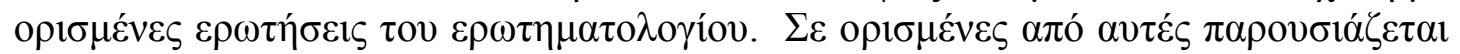

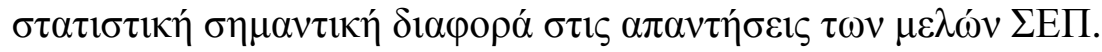

Пívaкas 6

А

\begin{tabular}{|c|c|c|c|c|}
\hline$\Delta \eta \lambda \hat{\sigma} \sigma \varepsilon 1 \varsigma$ & 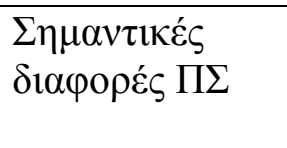 & \multicolumn{2}{|c|}{$\begin{array}{l}\text { Iean rank } \\
\text {-Kruskal- Wallis } \\
\text { test }\end{array}$} & p-value \\
\hline 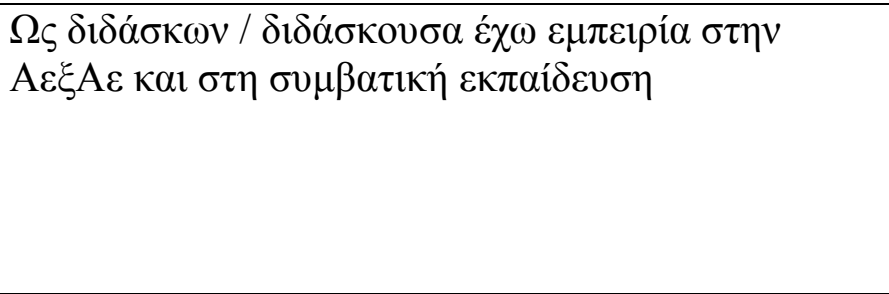 & 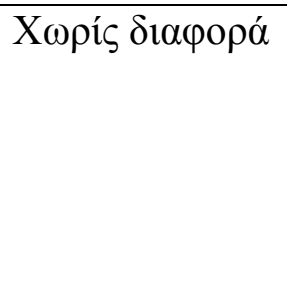 & $\begin{array}{l}\Delta \mathrm{MY} \\
\mathrm{E} \Lambda \Pi \mathrm{O} \\
\Lambda \\
\mathrm{E} \Pi \mathrm{A} \\
\Pi \Lambda \mathrm{H} \Sigma \\
\mathrm{M} \Delta \mathrm{E}\end{array}$ & $\begin{array}{l}36,90 \\
39,71 \\
32,58 \\
34,05 \\
45,56\end{array}$ & 0,193 \\
\hline 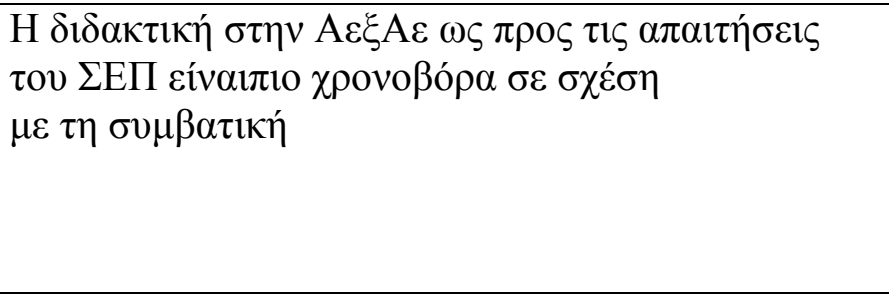 & 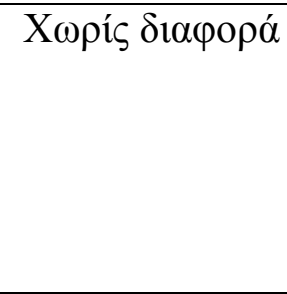 & $\begin{array}{l}\Delta \mathrm{MY} \\
\mathrm{E} \Lambda \Pi \mathrm{O} \\
\Lambda \\
\mathrm{E} \Pi \mathrm{A} \\
\Pi \Lambda \mathrm{H} \Sigma \\
\mathrm{M} \Delta \mathrm{E} \\
\end{array}$ & $\begin{array}{l}39,13 \\
31,34 \\
38,35 \\
44,50 \\
39,97\end{array}$ & 0,506 \\
\hline 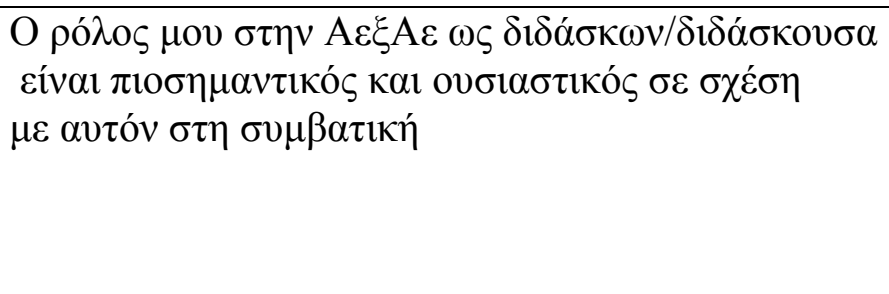 & 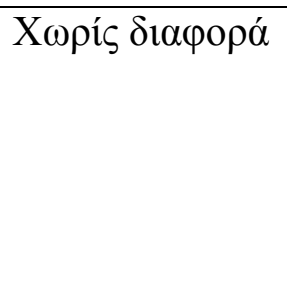 & $\begin{array}{l}\Delta \mathrm{MY} \\
\mathrm{E} \Lambda \Pi \mathrm{O} \\
\Lambda \\
\mathrm{E} \Pi \mathrm{A} \\
\Pi \Lambda \mathrm{H} \Sigma \\
\mathrm{M} \Delta \mathrm{E}\end{array}$ & $\begin{array}{l}46,13 \\
33,21 \\
38,96 \\
41,95 \\
35,28\end{array}$ & 0,413 \\
\hline
\end{tabular}




\begin{tabular}{|c|c|c|c|c|}
\hline 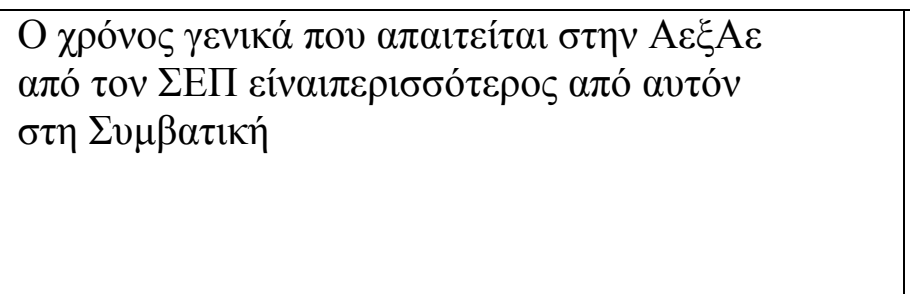 & 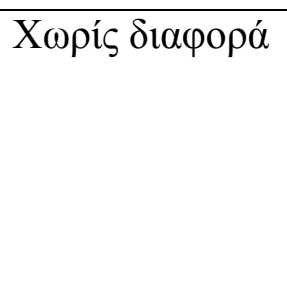 & $\begin{array}{l}\Delta \mathrm{MY} \\
\mathrm{E} \Lambda \Pi \mathrm{O} \\
\Lambda \\
\mathrm{E} \Pi \mathrm{A} \\
\Pi \Lambda \mathrm{H} \Sigma \\
\mathrm{M} \Delta \mathrm{E}\end{array}$ & $\begin{array}{l}43,00 \\
32,82 \\
39,62 \\
48,09 \\
34,08\end{array}$ & 0,277 \\
\hline 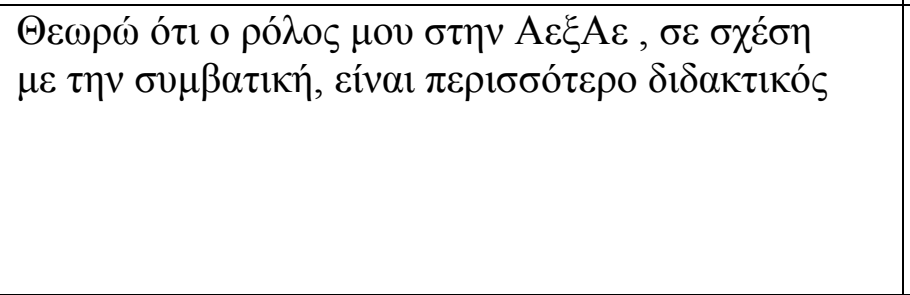 & X & $\begin{array}{l}\Delta \mathrm{MY} \\
\mathrm{E} \Lambda \Pi \mathrm{O} \\
\Lambda \\
\mathrm{E} \Pi \mathrm{A} \\
\Pi \Lambda \mathrm{H} \Sigma \\
\mathrm{M} \Delta \mathrm{E}\end{array}$ & $\begin{array}{l}36,30 \\
34,84 \\
31,88 \\
41,00 \\
43,94\end{array}$ & 0,505 \\
\hline 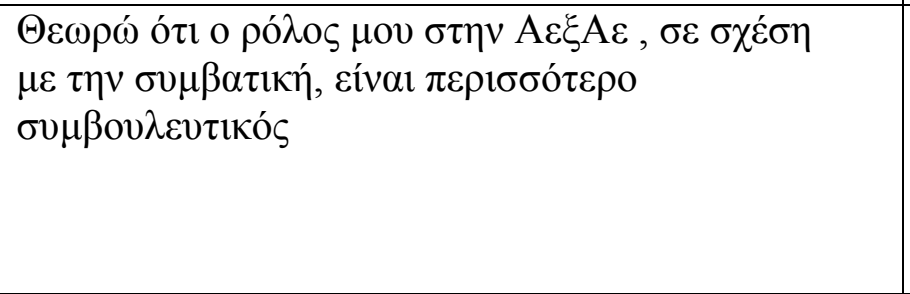 & $\begin{array}{l}\mathrm{M} \Delta \mathrm{E}>\Pi \Lambda \mathrm{H} \Sigma \\
\Delta \mathrm{MY}>\mathrm{E} \Lambda \Pi \mathrm{O} \Lambda \\
\mathrm{E} \Pi \mathrm{E}>\mathrm{E} \Lambda \Pi \mathrm{O} \Lambda \\
\mathrm{M} \Delta \mathrm{E}>\mathrm{E} \Lambda \Pi \mathrm{O} \Lambda\end{array}$ & $\begin{array}{l}\Delta \mathrm{MY} \\
\mathrm{E} \Lambda \Pi \mathrm{O} \\
\Lambda \\
\mathrm{E} \Pi \mathrm{A} \\
\Pi \Lambda \mathrm{H} \Sigma \\
\mathrm{M} \Delta \mathrm{E}\end{array}$ & $\begin{array}{l}44,40 \\
30,44 \\
40,77 \\
26,55 \\
45,22\end{array}$ & 0,033 \\
\hline 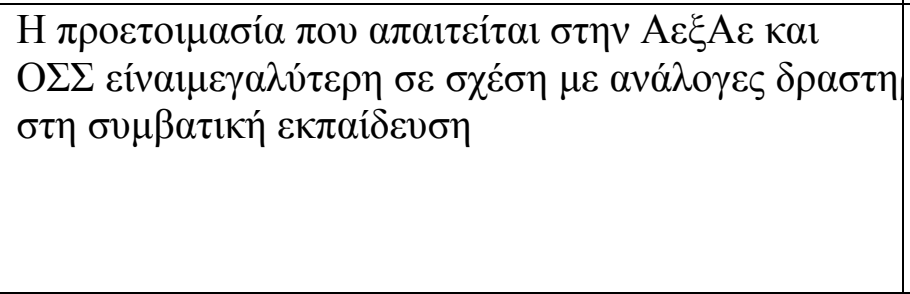 & 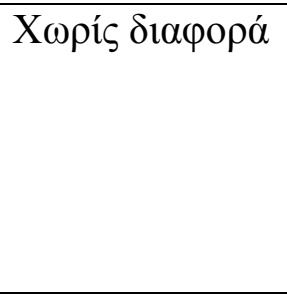 & $\begin{array}{l}\Delta \mathrm{MY} \\
\mathrm{E} \Lambda \Pi \mathrm{O} \\
\Lambda \\
\mathrm{E} \Pi \mathrm{A} \\
\Pi \Lambda \mathrm{H} \Sigma \\
\mathrm{M} \Delta \mathrm{E}\end{array}$ & $\begin{array}{l}36,30 \\
36,92 \\
31,69 \\
48,05 \\
37,44\end{array}$ & 0,441 \\
\hline 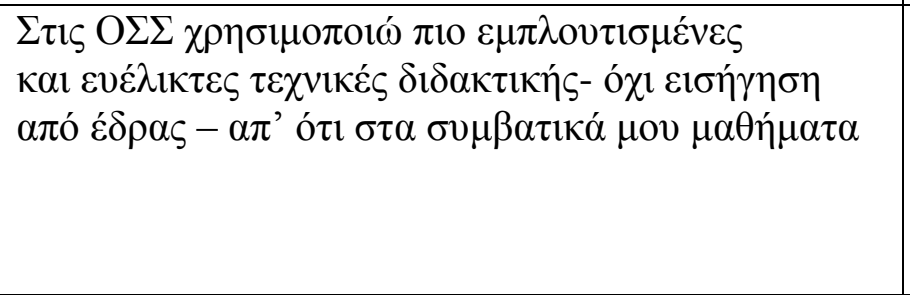 & 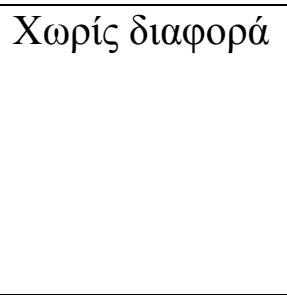 & $\begin{array}{l}\Delta \mathrm{MY} \\
\mathrm{E} \Lambda \Pi \mathrm{O} \\
\Lambda \\
\mathrm{E} \Pi \mathrm{A} \\
\Pi \Lambda \mathrm{H} \Sigma \\
\mathrm{M} \Delta \mathrm{E}\end{array}$ & $\begin{array}{l}39,43 \\
39,61 \\
36,73 \\
41,05 \\
36,28\end{array}$ & 0,967 \\
\hline 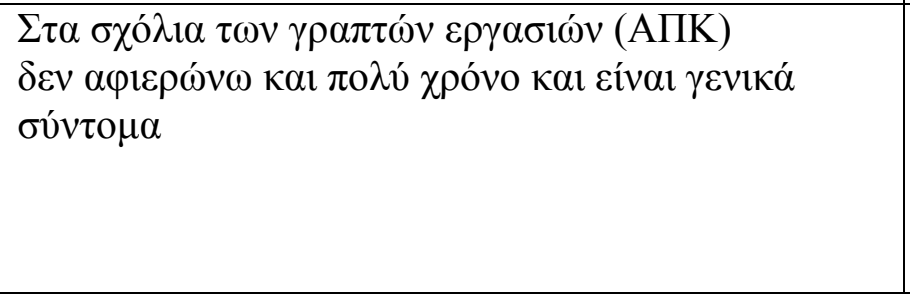 & $\begin{array}{l}\Pi \Lambda \mathrm{H} \Sigma>\mathrm{E} \Lambda \Pi \mathrm{O} \\
\Lambda \\
\mathrm{M} \triangle \mathrm{E}>\mathrm{E} \Lambda \Pi \mathrm{O} \Lambda\end{array}$ & $\begin{array}{l}\Delta \mathrm{MY} \\
\mathrm{E} \Lambda \Pi \mathrm{O} \\
\Lambda \\
\mathrm{E} \Pi \mathrm{A} \\
\Pi \Lambda \mathrm{H} \Sigma \\
\mathrm{M} \Delta \mathrm{E}\end{array}$ & $\begin{array}{l}30,57 \\
26,08 \\
37,04 \\
50,32 \\
52,06\end{array}$ & 0,000 \\
\hline 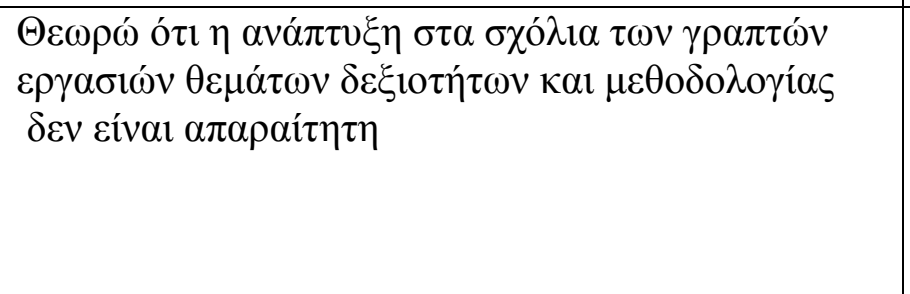 & $\begin{array}{l}\Pi \Lambda H \Sigma>\Delta M Y \\
\Pi \Lambda H \Sigma>E \Lambda \Pi O \\
\Lambda \\
\Pi \Lambda H \Sigma>\text { EПA } \\
\Pi \Lambda H \Sigma>M \Delta E\end{array}$ & $\begin{array}{l}\Delta \mathrm{MY} \\
\mathrm{E} \Lambda \Pi \mathrm{O} \\
\Lambda \\
\mathrm{E} \Pi \mathrm{A} \\
\Pi \Lambda \mathrm{H} \Sigma \\
\mathrm{M} \Delta \mathrm{E}\end{array}$ & $\begin{array}{l}32,63 \\
29,89 \\
35,04 \\
56,18 \\
44,17\end{array}$ & 0,005 \\
\hline 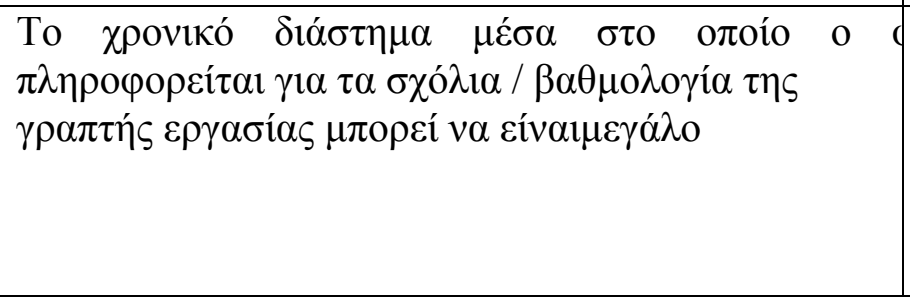 & 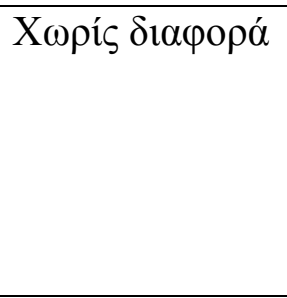 & $\begin{array}{l}\Delta \mathrm{MY} \\
\mathrm{E} \Lambda \Pi \mathrm{O} \\
\Lambda \\
\mathrm{E} \Pi \mathrm{A} \\
\Pi \Lambda \mathrm{H} \Sigma \\
\mathrm{M} \Delta \mathrm{E}\end{array}$ & $\begin{array}{l}35,50 \\
41,74 \\
36,88 \\
38,09 \\
39,00\end{array}$ & 0,934 \\
\hline
\end{tabular}




\begin{tabular}{|c|c|c|c|c|}
\hline 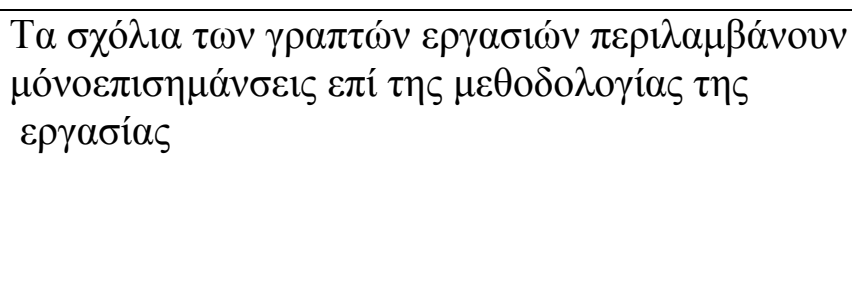 & $\begin{array}{l}\Pi \Lambda H \Sigma>\Delta \mathrm{MY} \\
\Pi \Lambda \mathrm{H} \Sigma>\mathrm{E} \Lambda \Pi \mathrm{O} \\
\Lambda\end{array}$ & $\begin{array}{l}\Delta \mathrm{MY} \\
\mathrm{E} \Lambda \Pi \mathrm{O} \\
\Lambda \\
\mathrm{E} \Pi \mathrm{A} \\
\Pi \Lambda \mathrm{H} \Sigma \\
\mathrm{M} \Delta \mathrm{E}\end{array}$ & $\begin{array}{l}31,77 \\
28,08 \\
40,23 \\
51,32 \\
46,03\end{array}$ & 0,007 \\
\hline 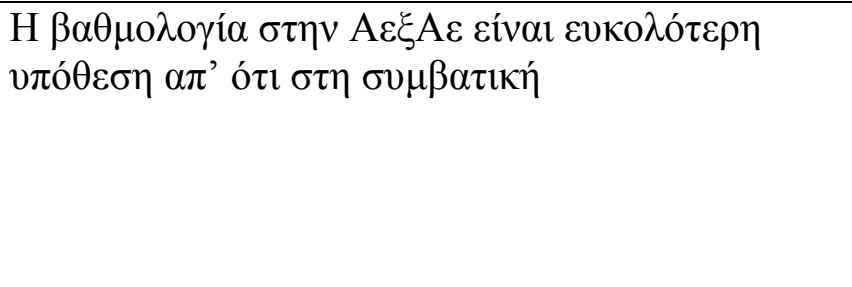 & 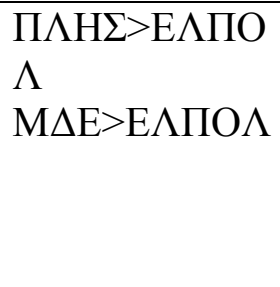 & $\begin{array}{l}\Delta \mathrm{MY} \\
\mathrm{E} \Lambda \Pi \mathrm{O} \\
\Lambda \\
\mathrm{E} \Pi \mathrm{A} \\
\Pi \Lambda \mathrm{H} \Sigma \\
\mathrm{M} \Delta \mathrm{E}\end{array}$ & $\begin{array}{l}42,00 \\
25,37 \\
36,00 \\
49,95 \\
44,25\end{array}$ & 0,010 \\
\hline
\end{tabular}

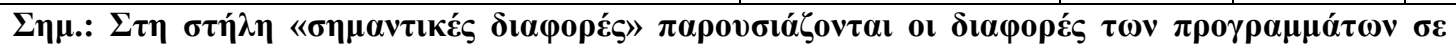

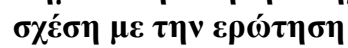

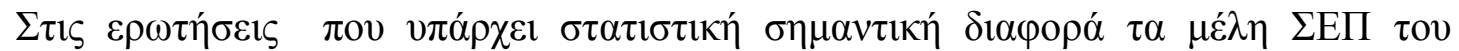

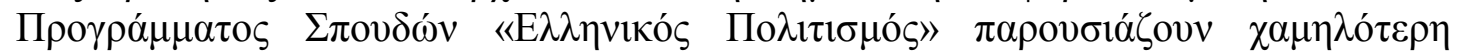

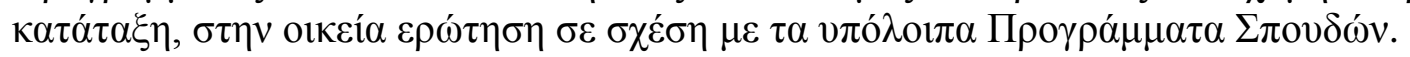

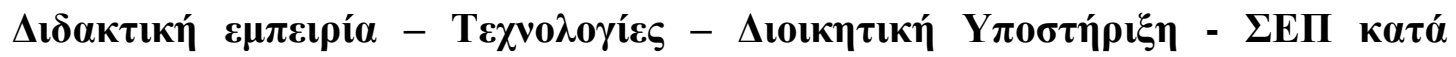

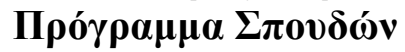

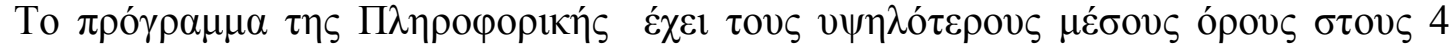

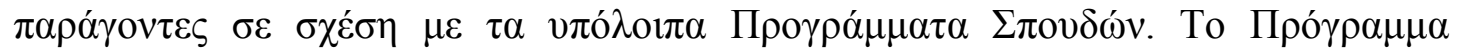

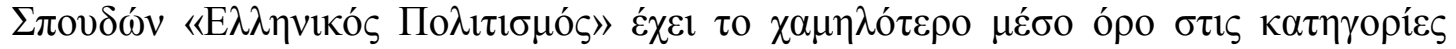

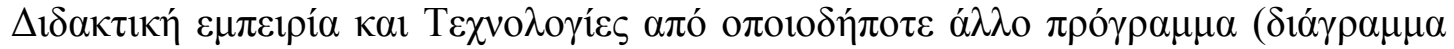
$1)$.

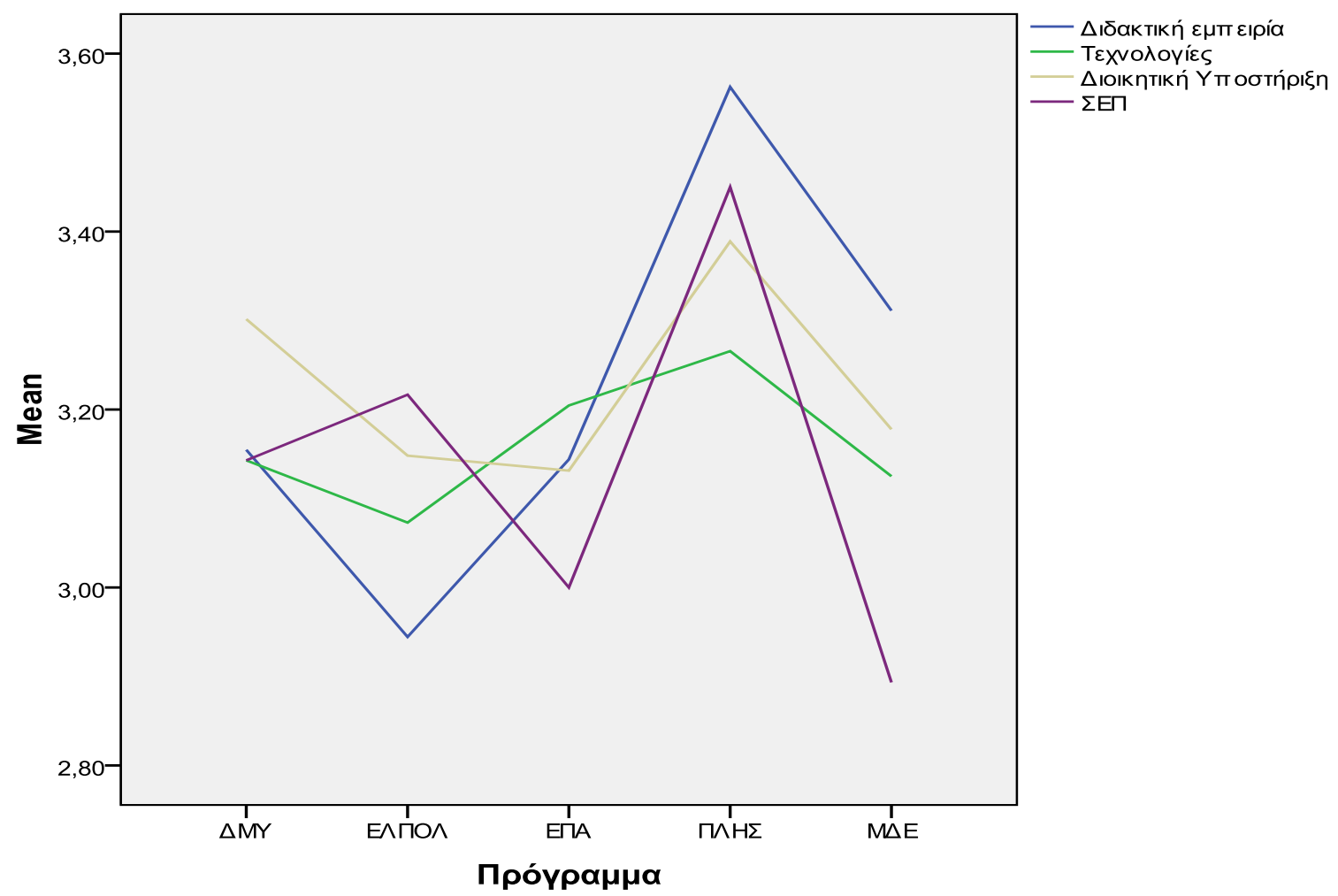

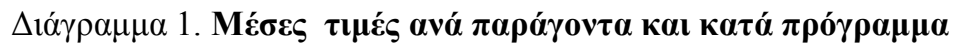




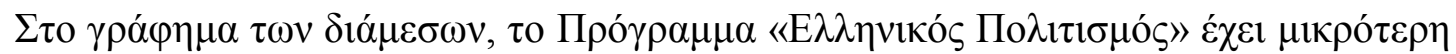

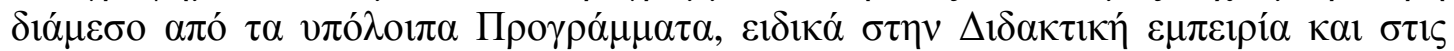

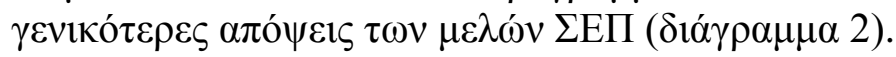

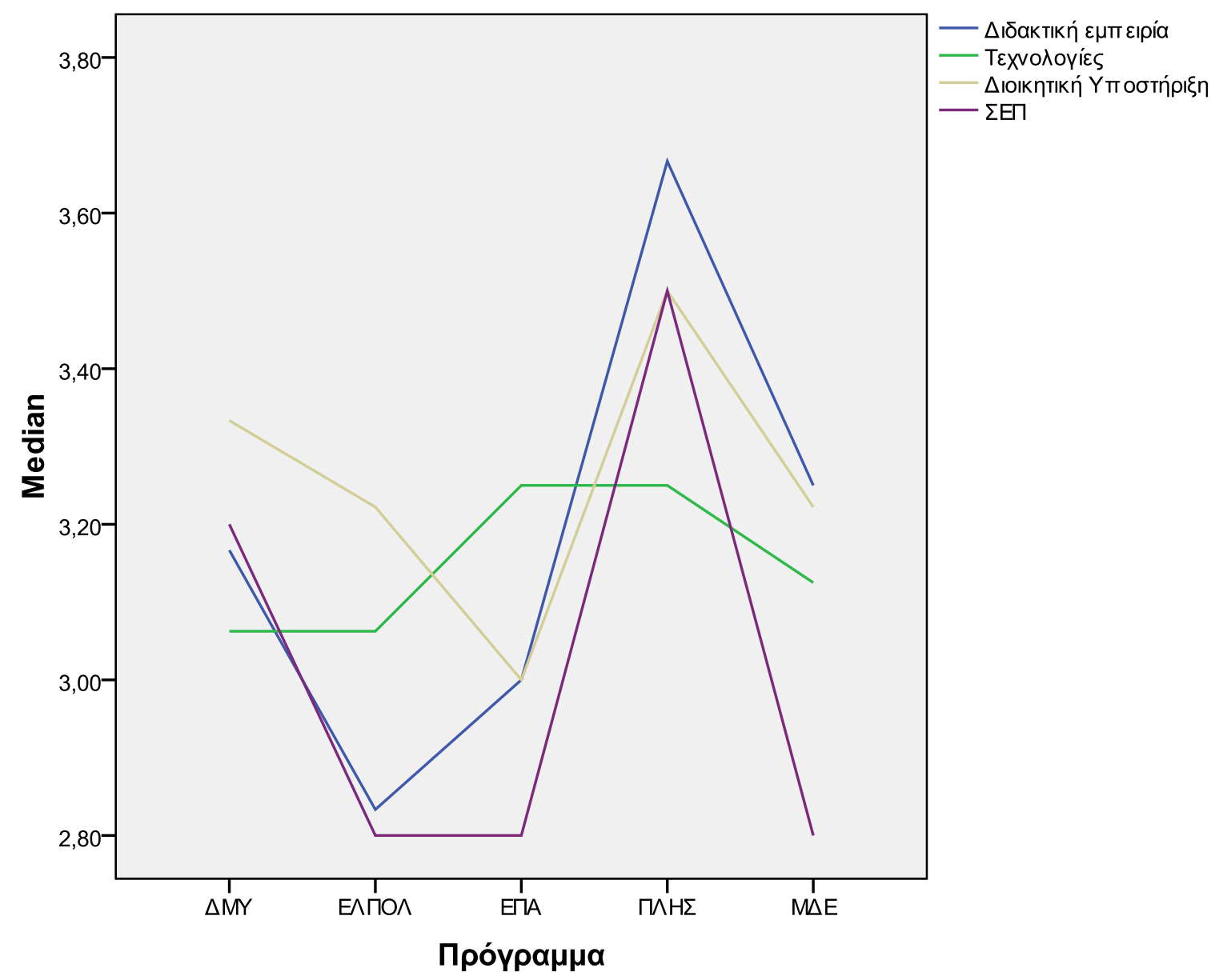

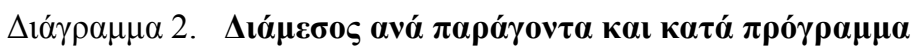

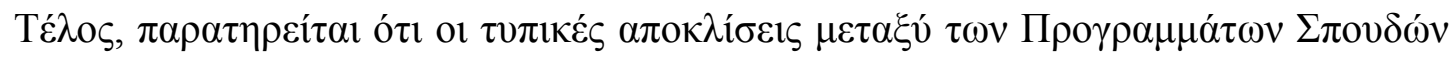

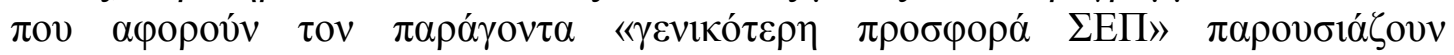

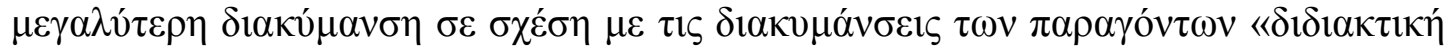

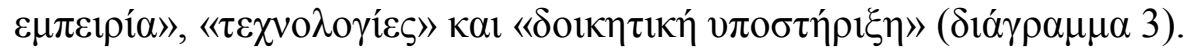




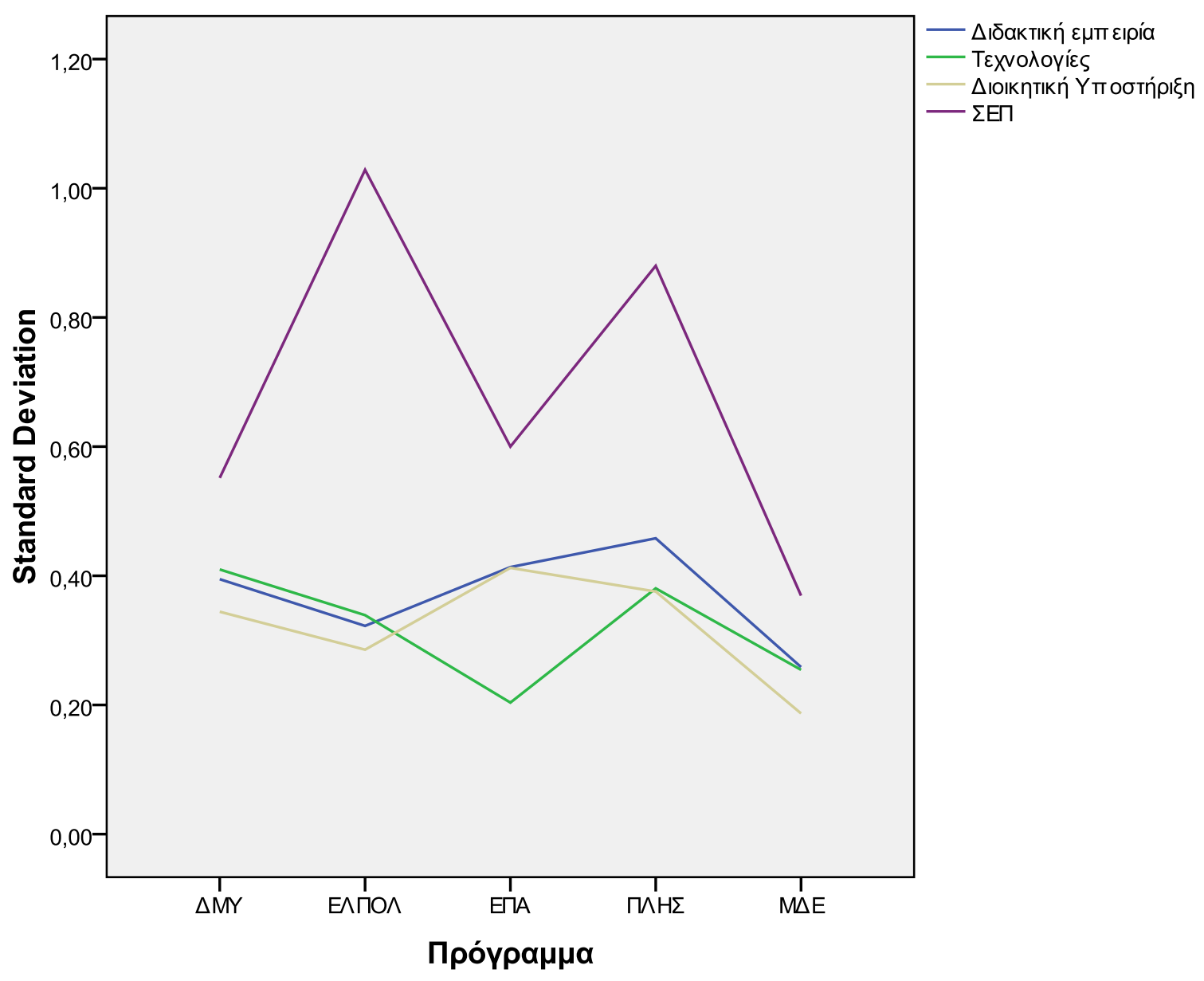

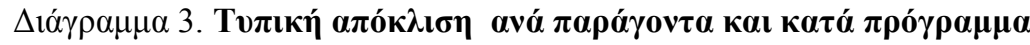

\section{$\Sigma$ YZHTH $\Sigma$ H - $\Sigma$ YMПЕРА $\Sigma$ MATA}

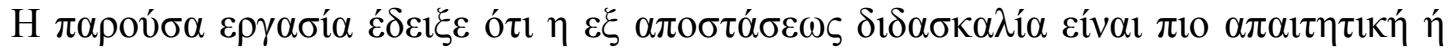

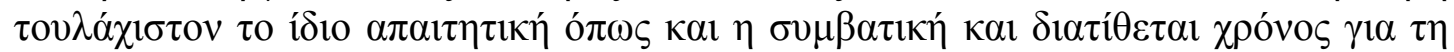

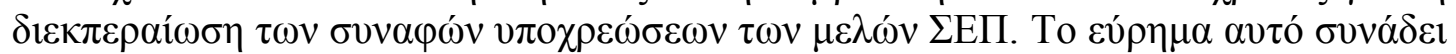

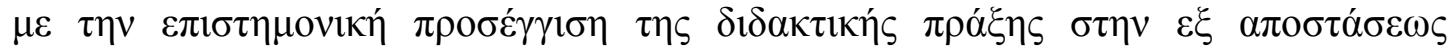

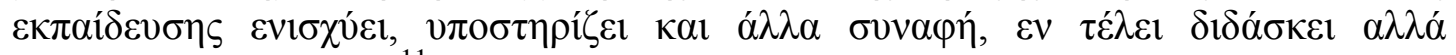

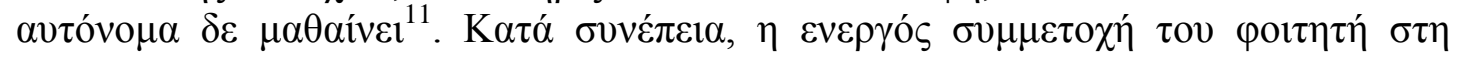

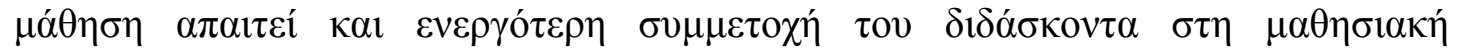
$\delta i \varepsilon \rho \gamma \alpha \sigma i ́ \alpha$.

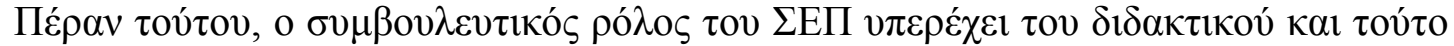

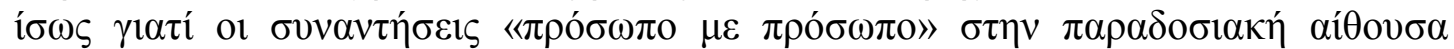

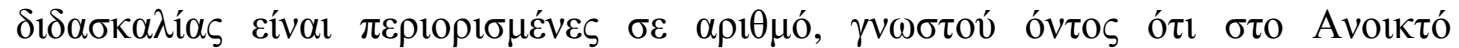

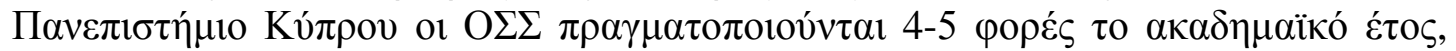

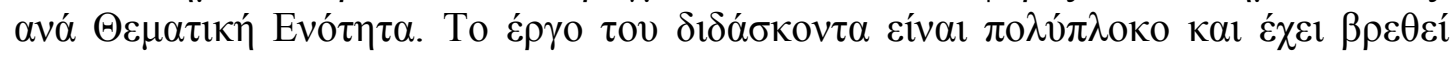

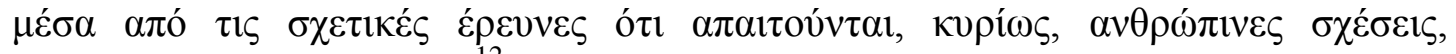
$\varepsilon \mu \psi v ́ \chi \omega \sigma \eta \kappa \alpha l \varepsilon \pi \imath \kappa o t v \omega v i ́ \alpha .{ }^{12}$

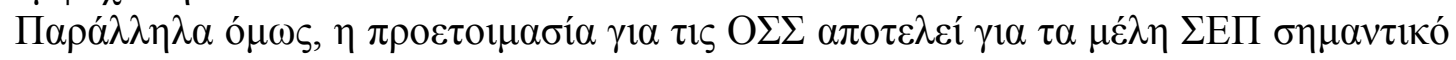

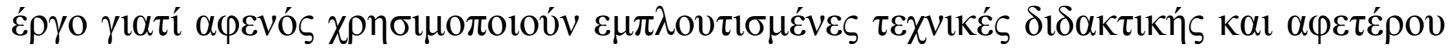

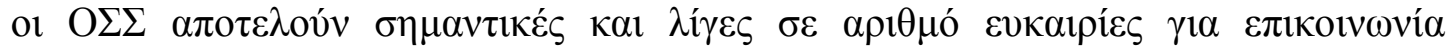

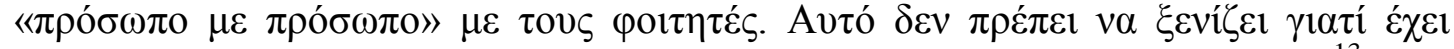

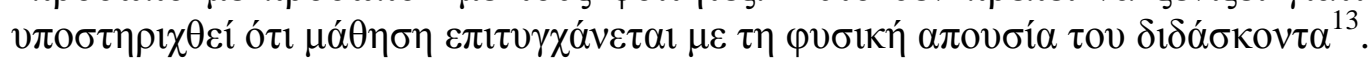




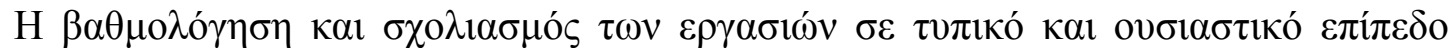

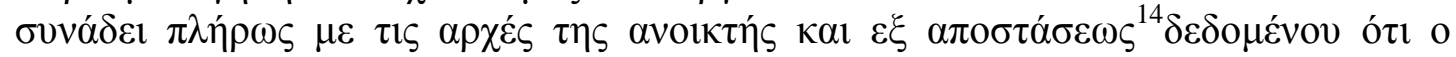

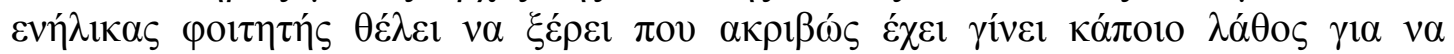

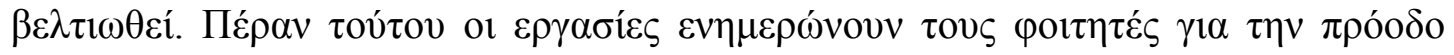

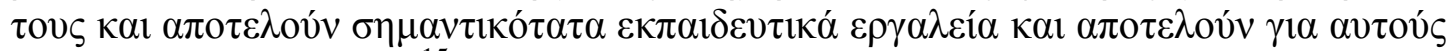

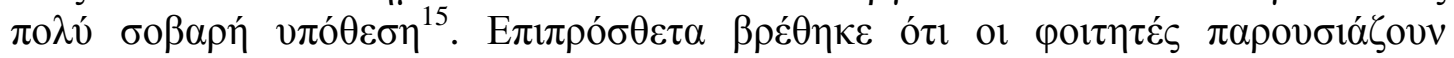

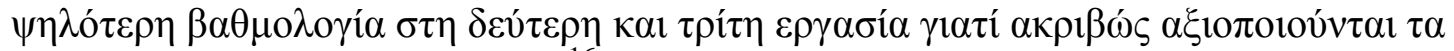

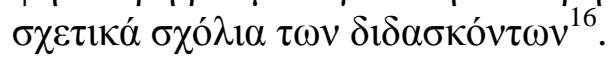

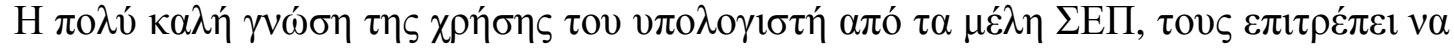

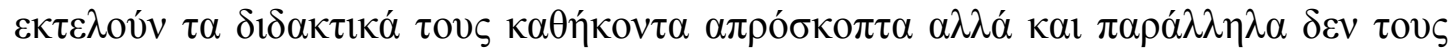

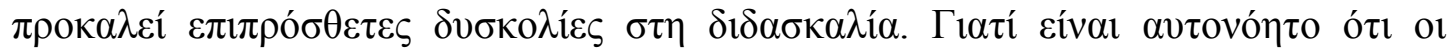

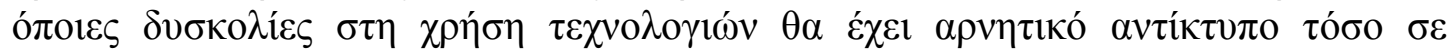

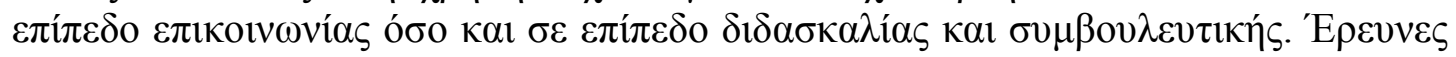

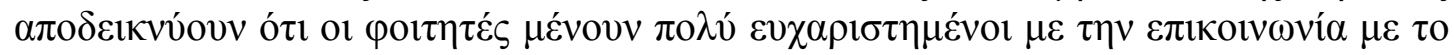

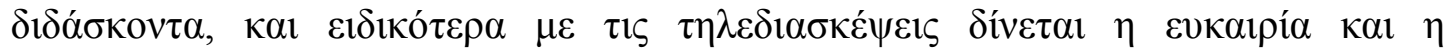

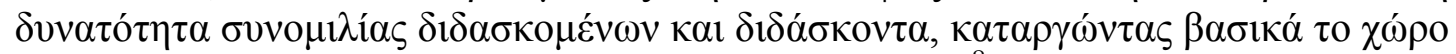

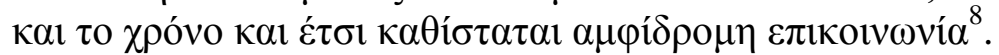

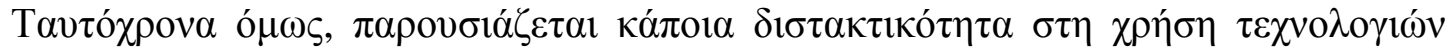

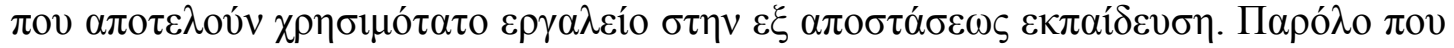

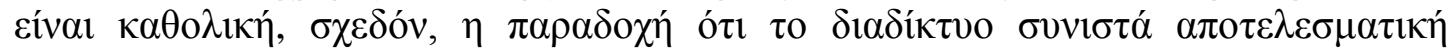

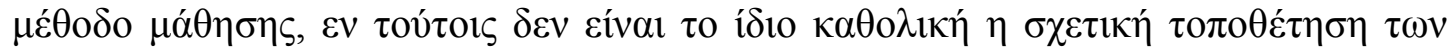

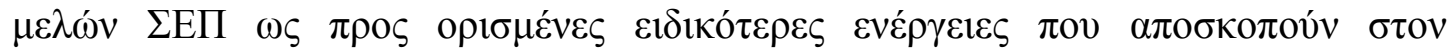

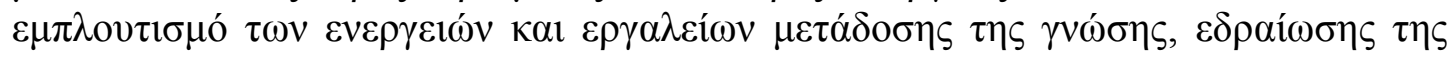

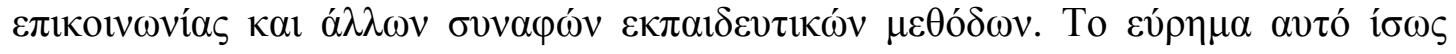

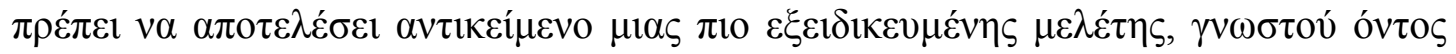

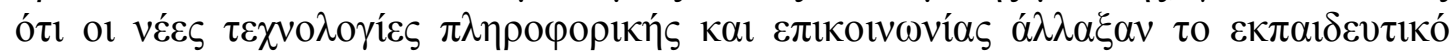
бкฤviкó.

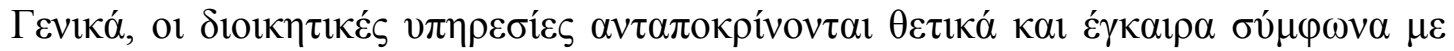

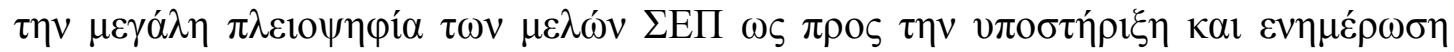

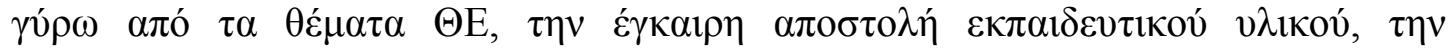

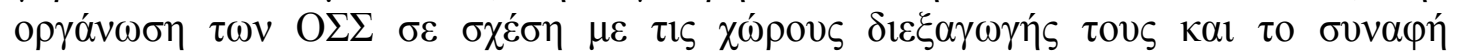

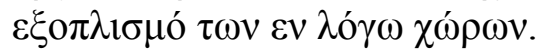

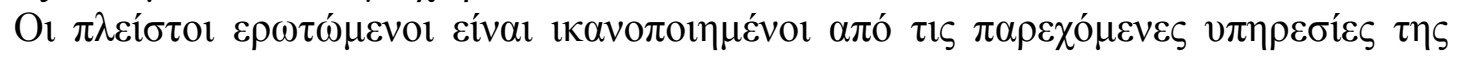
$\beta 1 \beta \lambda$ เо

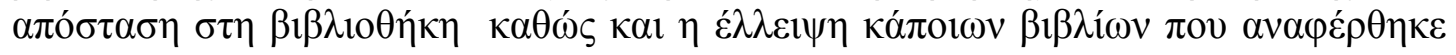

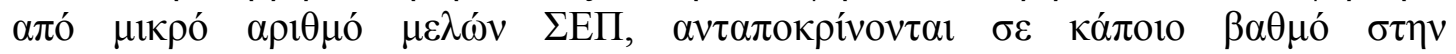

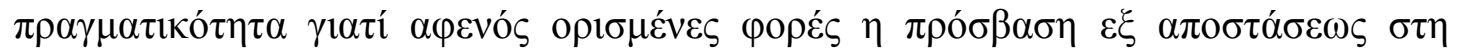

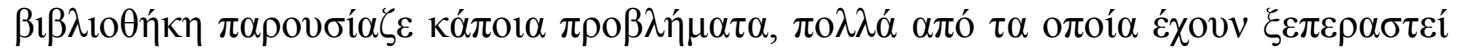

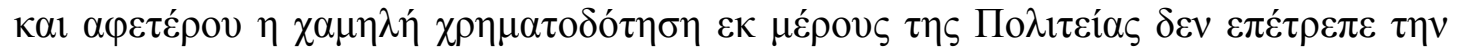

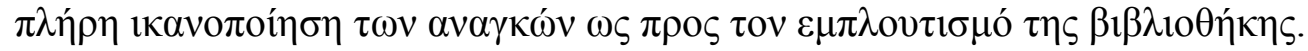

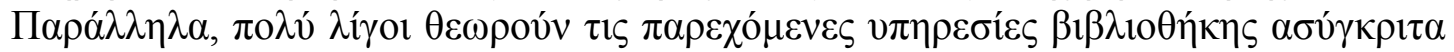

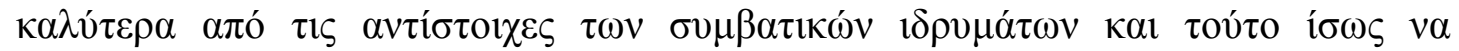

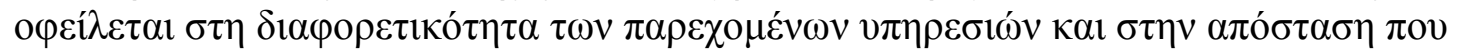

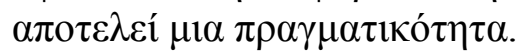

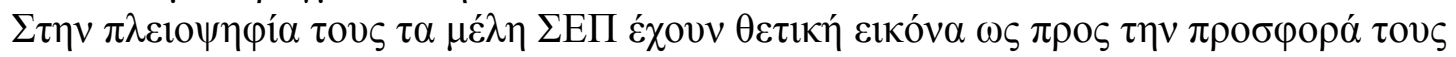

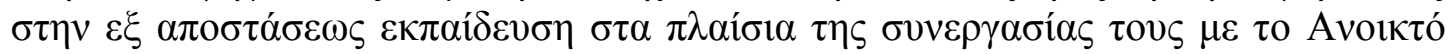

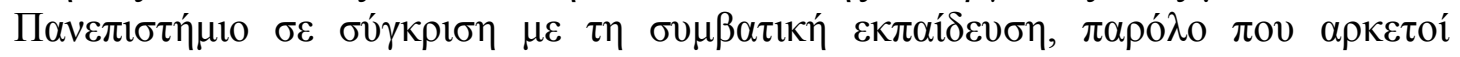

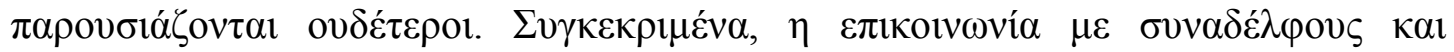

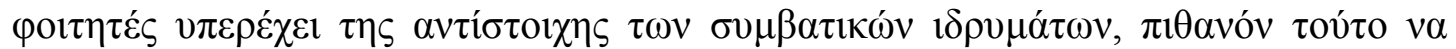

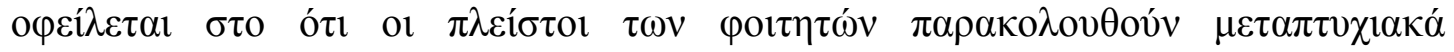




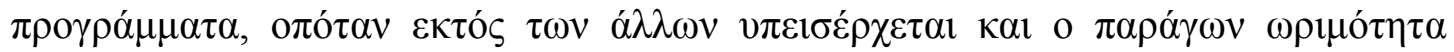

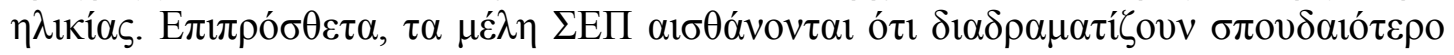

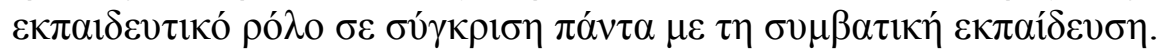

\section{ВIВ $\Lambda$ IОГРАФІА}

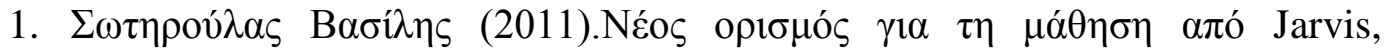

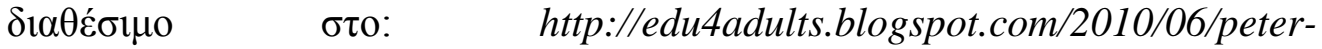
jarvis.html\#ixzz1LE9fovlN ( $\pi \rho o ́ \sigma \beta \alpha \sigma \eta 10 / 05 / 2011)$

2. European Commission (2001). Implementing a new open method of coordination. Bulletin EU3-2000, Conclusions of the Presidency (17/23)

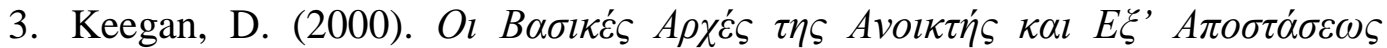

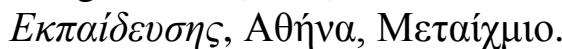

4. Modesto S.T. and Tau R. D. (2010) Introducing distance education, Virtual

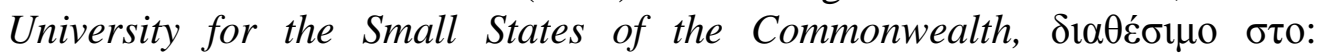
http://www.col.org/SiteCollectionDocuments/Introducing_Distance_Educatio $\underline{\text { n.pdf }}\left(\pi \rho \sigma_{\sigma} \sigma \beta \alpha \sigma \eta 22 / 05 / 2011\right)$

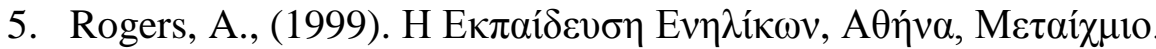

6. Gunawardena C., McIsaac S. M. (2001) Distanteducation, available at: www.aect.org/edtech/ed1/13/index.html ( $\pi \rho o ́ \sigma \beta \alpha \sigma \eta$ 22/05/2011)

7. Sammons, M. (1989). An epistemological justification for the role of teaching in distance education. The American Journal of Distance Education, 2(3), 516.

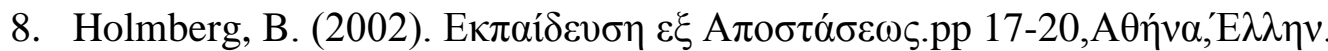

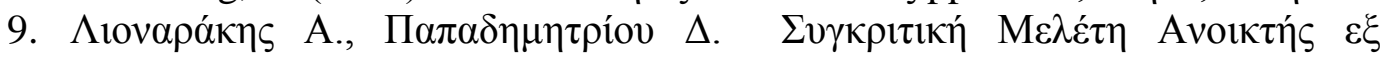

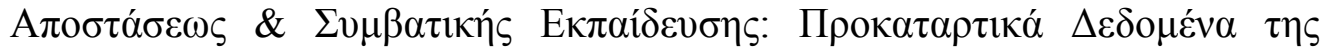

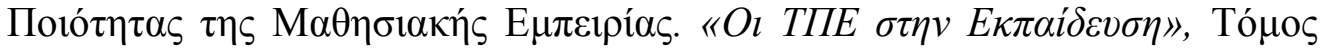

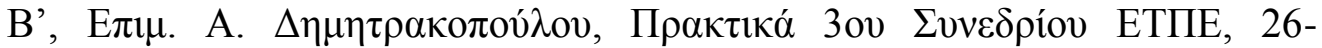

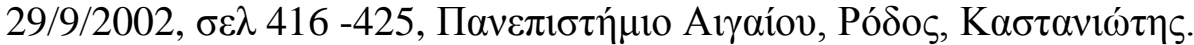

10. Howitt, D., \& Cramer, D. (1999). Computing Statistics with SPSS for Windows. pp136 -138 UK, Pearson Education Limited.

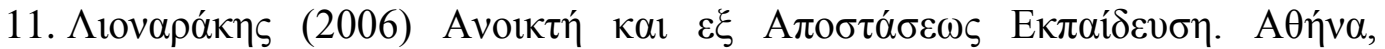
Пролонло́s.

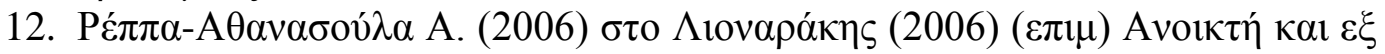

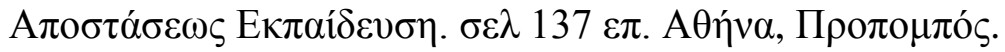

13. Rumble G. (1989) On defining distance education. The American Journal of

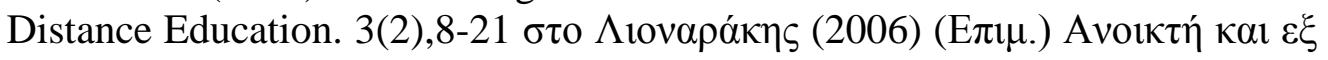

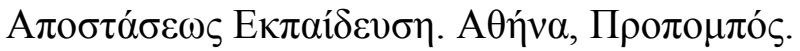

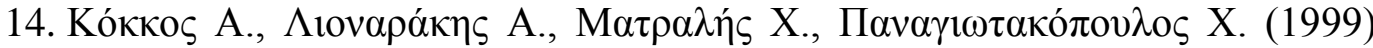

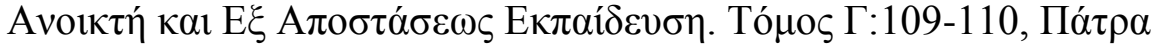

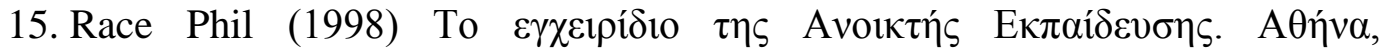

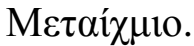

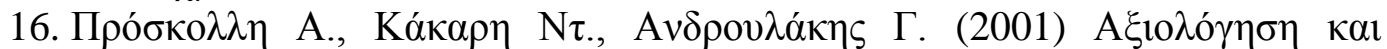

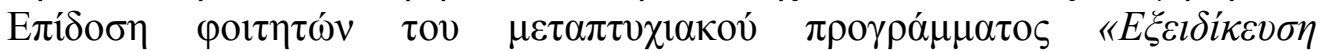

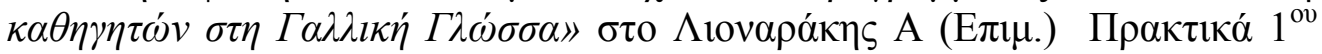

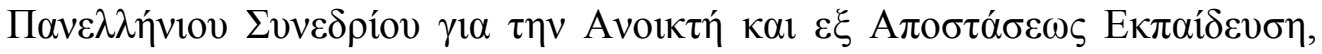

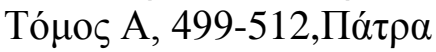

